Noname manuscript No.

(will be inserted by the editor)

\title{
Morphologically accurate reduced order modeling of spiking neurons
}

\author{
Anthony R. Kellems, Saifon Chaturantabut, \\ Danny C. Sorensen, and Steven J. Cox
}

Received: date / Accepted: date

\begin{abstract}
Accurately simulating neurons with realistic morphological structure and synaptic inputs requires the solution of large systems of nonlinear ordinary differential equations. We apply model reduction techniques to recover the complete nonlinear voltage dynamics of a neuron using a system of much lower dimension. Using a proper orthogonal decomposition, we build a reduced-order system from salient snapshots of the full system output, thus reducing the number of state variables. A discrete empirical interpolation method is then used to reduce the complexity of the nonlinear term to be proportional to the number of reduced variables. Together these two techniques allow for up to two orders of magnitude dimension reduction without sacrificing the spatially-distributed input structure, with an associated order of magnitude speed-up in simulation time. We demonstrate that both nonlinear spiking behavior and subthreshold response of realistic cells are accurately captured by these low-dimensional models.
\end{abstract}

Keywords Nonlinear model reduction · Hodgkin-Huxley · Proper orthogonal decomposition · Empirical interpolation method · Compartmental models

\section{Introduction}

Efforts to describe the interplay between morphology, kinetics, and synaptic inputs of single neurons are always, at their core, attempts at model reduction. For, all simulations require a balance between the realism of the model and the constraints of time and computing power. Investigators have developed neuronal models of varying levels of biophysical faithfulness, with the more detailed ones taking the form of compartmental models with voltage-gated ion channels. In order to resolve features at the level of dendritic spines $(1 \mu \mathrm{m})$, this can require many thousands of compartments, and to include realistic $\mathrm{Na}^{+}, \mathrm{K}^{+}$, and $\mathrm{Ca}^{2+}$ channel kinetics requires upwards of 10 variables

Anthony R. Kellems

Rice University

Tel.: (713)-348-3700

E-mail: tkellems@rice.edu 
per compartment. We thus arrive at a large, coupled, nonlinear system of differential equations which must be solved to obtain the cell's voltage dynamics.

Model reduction seeks to shrink the number of state variables while reproducing, as much as possible, the behaviors of the original model. Techniques to simplify the kinetics have resulted in approximations which use fewer gating variables, such as the method of equivalent potentials (Kepler et al., 1992). While such techniques were developed for single-compartment models, the kinetic schemes can be readily integrated into multicompartment models. Other techniques aim to simplify the morphology of the neuron in order to reduce the number of compartments and thus more drastically reduce the computational load. The first attempts at this were made by Wilfrid Rall. Using passive membrane models and morphologies satisfying the " $3 / 2$ " power law, he was able to rigorously show that symmetric synaptic inputs can be mapped to a single passive cable (Rall, 1959). However, his model assumptions were too strict for general use, and did not apply to cells with active kinetic properties, which are necessary to investigate realistic neuronal functions.

Investigators have since used other ad hoc techniques to achieve their model reduction goals. The 19-compartment reduction of hippocampal cells by (Traub and Miles, 1991) and the subsequent 2-compartment reduction by (Pinsky and Rinzel, 1994) have demonstrated that certain properties can be retained in such simple models by lumping many compartments together. However, this type of reduction sacrifices the spatial location of synapses. Previously, we demonstrated that we can preserve the spatial input-output relationship in a reduced neuronal model if we are content to reproduce only the subthreshold dynamics by considering the quasi-active version of the full model (Kellems et al., 2009).

Here we extend the results of our previous work to reproduce the full nonlinear behavior of morphologically accurate models by applying two model reduction techniques. The first reduces the number of state variables, while the second reduces the complexity of the nonlinear term by interpolating at a small number of dendritic locations. These techniques preserve the spatial precision of synaptic input while reproducing the global voltage dynamics, including both subthreshold and spiking behaviors.

We start in $\S 2$ with a description of the full model and the solution methods we employ. In $\S 3$ we apply the model reduction techniques to simulation data to arrive at the reduced system. Using this framework, in $\S 4$ we examine the accuracy of the reduced system on simplified morphologies and discuss challenges that branched cells pose. We promptly introduce algorithms to tackle these challenges, and show that they succeed for simple branched cells. In $§ 5-6$ we show that these techniques accurately reproduce the spiking dynamics of a broad class of realistic cells. We end with a discussion of applications, improvements, and extensions in $\S 7$.

\section{Nonlinear Cable Equation}

We consider dendritic neurons (see, e.g., Figure 1) with $D$ branched dendrites meeting at the soma. The $d$ th dendrite possesses $B_{d}$ branches, and we denote by $\ell_{b}$ the length of branch $b$ and encode its radius, as a function of distance from its distal end, by $a_{b}(x)$. The transmembrane potential along branch $b$ will be denoted by $v_{b}(x, t)$. We assume that the axial resistivity, $R_{i}(\mathrm{k} \Omega \mathrm{cm})$, and membrane capacitance, $C_{m}\left(\mu \mathrm{F} / \mathrm{cm}^{2}\right)$, are uniform throughout the cell. We suppose that branch $b$ carries $C$ distinct currents, with associated densities, $G_{b c}(x)\left(\mathrm{mS} / \mathrm{cm}^{2}\right)$ and reversal potentials $E_{c}, c=1, \ldots, C$. The 


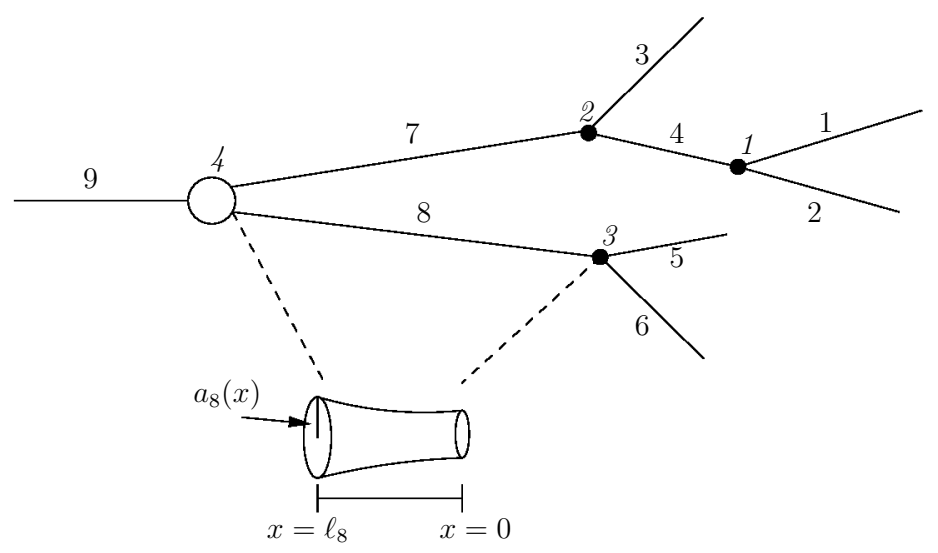

Fig. 1 Example of a simplified neuron to demonstrate our labeling scheme. Branches are indexed using the Hines ordering (Hines, 1984), and junctions are labeled with italics. For this cell there are $D=3$ dendrites and $J=4$ junctions. Our labeling scheme implies, for example, that at junction 3 the children are indexed by $b_{3}^{1}=5, b_{3}^{2}=6$ and the mother is indexed by $b_{3}^{3}=8$

kinetics of current $c$ on branch $b$ are governed by (powers of) the $F_{c}$ gating variables, $w_{b c f}, f=1, \ldots, F_{c}$. When subjected to input at $S_{b}$ synapses, these gating variables, together with $v_{b}$, obey the nonlinear cable equation

$$
\begin{gathered}
a_{b} C_{m} \partial_{t} v_{b}=\frac{1}{2 R_{i}} \partial_{x}\left(a_{b}^{2} \partial_{x} v_{b}\right)-a_{b} \sum_{c=1}^{C} G_{b c}(x)\left(v_{b}-E_{c}\right) \prod_{f=1}^{F_{c}} w_{b c f}^{q_{c f}} \\
-\frac{1}{2 \pi} \sum_{s=1}^{S_{b}} g_{b s}(t) \delta\left(x-x_{b s}\right)\left(v_{b}-E_{b s}\right) \\
\partial_{t} w_{b c f}=\frac{w_{c f, \infty}\left(v_{b}\right)-w_{b c f}}{\tau_{c f}\left(v_{b}\right)}, \quad 0<x<\ell_{b}, \quad 0<t .
\end{gathered}
$$

Here $g_{b s}(\mathrm{nS})$ is the time course, $x_{b s}$ is the spatial location, and $E_{b s}$ is the reveral potential of the $s$ th synapse on branch $b$.

These branch potentials interact at $J$ junction points, where junction $J$ denotes the soma. At junction $j<J$ we denote by $b_{j}^{1}$ and $b_{j}^{2}$ the branch indices of the two children and by $b_{j}^{3}$ the branch index of the mother. Continuity of the potential at each junction requires

$$
v_{b_{j}^{1}}\left(\ell_{b_{j}^{1}}, t\right)=v_{b_{j}^{2}}\left(\ell_{b_{j}^{2}}, t\right)=v_{b_{j}^{3}}(0, t), \quad j=1, \ldots, J-1
$$

while current balance there requires

$$
a_{b_{j}^{1}}^{2}\left(\ell_{b_{j}^{1}}\right) \partial_{x} v_{b_{j}^{1}}\left(\ell_{b_{j}^{1}}, t\right)+a_{b_{j}^{2}}^{2}\left(\ell_{b_{j}^{2}}\right) \partial_{x} v_{b_{j}^{2}}\left(\ell_{b_{j}^{2}}, t\right)=a_{b_{j}^{3}}^{2}(0) \partial_{x} v_{b_{j}^{3}}(0, t), \quad j=1, \ldots, J-1 .
$$

The $D$ dendrites join at the soma and associated branch indices are $b_{J}^{d}, d=1, \ldots, D$. Continuity of the potential at the soma then reads

$$
v_{b_{J}^{1}}\left(\ell_{b_{J}^{1}}, t\right)=v_{b_{J}^{d}}\left(\ell_{b_{J}^{d}}, t\right), \quad d=2, \ldots, D
$$


we denote this common value by $v_{\sigma}(t)$ and note that current balance there requires

$$
\begin{aligned}
C_{m} \partial_{t} v_{\sigma}(t)= & \frac{\pi}{A_{\sigma} R_{i}} \sum_{d=1}^{D} a_{b_{J}^{d}}^{2}\left(\ell_{b_{J}^{d}}\right) \partial_{x} v_{b_{J}^{d}}\left(\ell_{b_{J}^{d}}, t\right)-\sum_{c=1}^{C} G_{\sigma c}\left(v_{\sigma}(t)-E_{c}\right) \prod_{f=1}^{F_{c}} w_{\sigma c f}^{q_{c f}}(t) \\
& -\frac{1}{A_{\sigma}} \sum_{s=1}^{S_{\sigma}} g_{\sigma s}(t)\left(v_{\sigma}(t)-E_{\sigma s}\right) \\
\partial_{t} w_{\sigma c f}(t)= & \frac{w_{c f, \infty}\left(v_{\sigma}(t)\right)-w_{\sigma c f}(t)}{\tau_{c f}\left(v_{\sigma}(t)\right)}, \quad 0<x<\ell_{b}, \quad 0<t .
\end{aligned}
$$

where $\sigma$ is the somatic index and $A_{\sigma}$ is the surface area of the soma. Finally, we denote by $\mathcal{L}$ the set of leaf indices, where a leaf is a branch with no children. We suppose that each leaf is sealed at its distal end, i.e.,

$$
\partial_{x} v_{b}(0, t)=0, \quad b \in \mathcal{L} .
$$

Initially the neuron is at rest, implying that $\partial_{t} v_{b}(x, 0)=0$. We solve for the rest state and denote it by $\bar{v}_{b}(x)$, and similarly for the gating variables, which yields the initial conditions

$$
\begin{aligned}
v_{b}(x, 0) & =\bar{v}_{b}(x) \\
w_{b c f}(x, 0) & =\bar{w}_{b c f}(x) \equiv w_{c f, \infty}\left(\bar{v}_{b}(x)\right) .
\end{aligned}
$$

With the rest state defined, it is easy to modify (1) to use current injection instead of synaptic conductance. If we substitute the rest state $\bar{v}_{b}(x)$ for the $v_{b}(x)$ in the synaptic input term, then this is equivalent to directly injecting current into the cell, which yields

$$
I_{\mathrm{inj}, b}(t) \equiv \frac{1}{2 \pi} \sum_{s=1}^{S_{b}} g_{b s}(t) \delta\left(x-x_{b s}\right)\left(\bar{v}_{b}(x)-E_{b s}\right) .
$$

If we now partition the cell into $N$ compartments, with $C$ currents per compartment and at most $F$ gating variables per current, then we arrive at the following system of ordinary differential equations

$$
\begin{aligned}
& \mathrm{v}^{\prime}(t)=\operatorname{Hv}(t)-\Phi(\mathrm{w}(t))\left(\operatorname{e} \cdot \mathrm{v}(t)-\mathrm{E}_{i}\right)+\mathrm{G}(t) \cdot\left(\mathrm{v}(t)-\mathrm{E}_{s}\right), \quad \mathrm{v}(t) \in \mathbf{R}^{N} \\
& \mathrm{w}^{\prime}(t)=(\mathrm{A}(\mathrm{v}(t))-\mathrm{w}(t)) \cdot / \mathrm{B}(\mathrm{v}(t)), \quad \mathrm{w}(t) \in \mathbf{R}^{N \times C \times F}
\end{aligned}
$$

where $\mathrm{H}$ is the $N$-by- $N$ Hines matrix, e $=\left[\begin{array}{llll}1 & 1 & \cdots & 1\end{array}\right]^{T} \in \mathbf{R}^{C}$,

$$
\begin{aligned}
& \Phi: \mathbf{R}^{N \times C \times F} \rightarrow \mathbf{R}^{N \times C}, \quad \mathrm{G}: \mathbf{R} \rightarrow \mathbf{R}^{N}, \\
& \mathrm{~A}: \mathbf{R}^{N} \rightarrow \mathbf{R}^{N \times C \times F} \text { and } \quad \mathrm{B}: \mathbf{R}^{N} \rightarrow \mathbf{R}^{N \times C \times F},
\end{aligned}
$$

$\mathrm{E}_{i}$ is the vector of channel reversal potentials

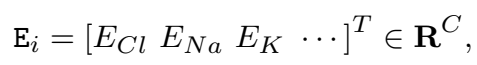

$\mathrm{E}_{s}$ is the vector of synaptic reversal potentials

$$
\mathrm{E}_{s}=\left[\begin{array}{llll}
E_{e x} & E_{e x} & E_{i n} & \cdots
\end{array}\right]^{T} \in \mathbf{R}^{N},
$$


and the 'dot' operator, a.b, denotes element-wise multiplication. In this paper, we use only excitatory synapses with $E_{e x}=0 \mathrm{mV}$. Here A and B are pointwise functions whose actions are defined, recall (2), by

$$
\mathrm{A}(\mathrm{v})_{i}=w_{:, \infty}\left(\mathrm{v}_{i}\right), \quad \mathrm{B}(\mathrm{v})_{i}=\tau_{:}\left(\mathrm{v}_{i}\right), \quad i=1, \ldots, N .
$$

We discretize (12)-(13) following the second-order staggered Euler scheme of (Hines, 1984). More precisely, for a fixed timestep $\Delta t$, we evaluate

$\mathrm{G}^{(j)}=\mathrm{G}((j-3 / 2) \Delta t), \quad \mathrm{w}^{(j)} \approx \mathrm{w}((j-3 / 2) \Delta t) \quad$ and $\quad \mathrm{v}^{(j)} \approx \mathrm{v}((j-1) \Delta t), \quad j=1,2, \ldots$

via the marching scheme: Given $\mathrm{w}^{(j-1)}$ and $\mathrm{v}^{(j-1)}$, evaluate

$$
\mathrm{w}^{(j)}=\left[\left(2 \mathrm{~B}\left(\mathrm{v}^{(j-1)}\right)-\Delta t\right) \cdot \mathrm{w}^{(j-1)}+2 \mathrm{~A}\left(\mathrm{v}^{(j-1)}\right) \Delta t\right] \cdot /\left[2 \mathrm{~B}\left(v^{(j-1)}\right)+\Delta t\right]
$$

and

$$
\mathrm{v}^{(j)}=2 \mathrm{v}_{\text {mid }}-\mathrm{v}^{(j-1)}
$$

where $\mathrm{v}_{\text {mid }}$ is the solution to the linear system

$$
\left(2 I / \Delta t-\mathrm{H}+\Phi\left(\mathrm{w}^{(j)}\right) \mathrm{e} .+\mathrm{G}^{(j)} \cdot\right) \mathrm{v}_{\text {mid }}=2 \mathrm{v}^{(j-1)} / \Delta t+\Phi\left(\mathrm{w}^{(j)}\right) \mathrm{E}_{i}+\mathrm{G}^{(j)} \cdot \mathrm{E}_{s} .
$$

In the case of current injection, $\mathrm{v}_{\text {mid }}$ is the solution to the linear system

$$
\left(2 I / \Delta t-\mathrm{H}+\Phi\left(\mathrm{w}^{(j)}\right) \mathrm{e} .\right) \mathrm{v}_{\operatorname{mid}}=2 \mathrm{v}^{(j-1)} / \Delta t+\Phi\left(\mathrm{w}^{(j)}\right) \mathrm{E}_{i}-\mathrm{G}^{(j)} \cdot\left(\overline{\mathrm{v}}-\mathrm{E}_{s}\right),
$$

where $\bar{v}$ is the rest potential of the discretized system.

\section{The Reduced Cable Equation}

We apply two model reduction techniques to this ODE system, both of which use the proper orthogonal decomposition (POD). The first technique generates a lowdimensional basis for the state variables, $\mathrm{v}$ and $\mathrm{w}$, while the second generates a lowdimensional basis for the nonlinear term.

\subsection{Proper Orthogonal Decomposition}

Given that $\mathrm{v} \in \mathbf{R}^{N}$, we wish to find a subspace $\mathcal{U} \subset \mathbf{R}^{N}$ of dimension $k \ll N$ in which the relevant states $\mathrm{v}$ are nearly contained. Specifically, given $n$ "snapshots" of the state variables $\mathcal{X}=\left[\xi\left(t_{1}\right) \xi\left(t_{2}\right) \cdots \xi\left(t_{n}\right)\right]$, we wish to find an orthonormal basis $\left\{\phi_{i}\right\}_{i=1}^{k} \subset \mathbf{R}^{N}$ which solves the minimization problem

$$
\min _{\left\{\phi_{i}\right\}_{i=1}^{k}} \sum_{j=1}^{n}\left\|\xi\left(t_{j}\right)-\sum_{i=1}^{k}\left(\xi\left(t_{j}\right)^{T} \phi_{i}\right) \phi_{i}\right\|_{2}^{2}
$$

i.e., we desire the $k$-dimensional basis which best fits, in the least squares sense, the snapshot data (Kunisch and Volkwein, 2002). The proper orthogonal decomposition (POD) provides a solution to this problem via the singular value decomposition (SVD) (Liang et al., 2002). 
To obtain the POD basis we first take "snapshots" of the voltage and nonlinear terms at specific (usually equally-spaced) time points during the simulation. For convenience we denote the nonlinear term by

$$
\mathrm{N}(\mathrm{v}(t), \mathrm{w}(t)) \equiv \Phi(\mathrm{w}(t))\left(\mathrm{e} \cdot \mathrm{v}(t)-\mathrm{E}_{i}\right) .
$$

We save the values of $\mathrm{v}$ and $\mathrm{N}(\mathrm{v}, \mathrm{w})$ at times $t_{1}, t_{2}, \ldots, t_{n}$, where $t_{j}=j \Delta t$. The snapshots are stored column-wise in matrices

$$
\begin{aligned}
\mathrm{v} & =\left[\begin{array}{lll}
\mathrm{v}\left(t_{1}\right) \mathrm{v}\left(t_{2}\right) & \cdots & \mathrm{v}\left(t_{n}\right)
\end{array}\right] \\
\mathrm{F} & =\left[\begin{array}{llll}
\mathrm{N}\left(\mathrm{v}\left(t_{1}\right), \mathrm{w}\left(t_{1}\right)\right) & \mathrm{N}\left(\mathrm{v}\left(t_{2}\right), \mathrm{w}\left(t_{2}\right)\right) & \cdots & \mathrm{N}\left(\mathrm{v}\left(t_{n}\right), \mathrm{w}\left(t_{n}\right)\right)
\end{array}\right]
\end{aligned}
$$

The matrix $\mathrm{V} \in \mathbf{R}^{N \times n}$ will be used to build the POD basis, while $\mathrm{F} \in \mathbf{R}^{N \times n}$ will be used in $\S 3.2$.

We begin with the SVD's of the snapshot matrices

$$
\mathrm{V}=U \Sigma X^{T}, \quad \mathrm{~F}=W \Lambda Y^{T},
$$

where $U^{T} U=I, X^{T} X=I, W^{T} W=I, Y^{T} Y=I$, and $\Sigma$ and $\Lambda$ are diagonal and non-negative. These diagonal elements are ordered in a descending fashion. We choose $k_{v} \leq n$ and $k_{f} \leq n$, set

$$
\mathrm{U}=U\left(:, 1: k_{v}\right) \quad \text { and } \quad \mathrm{W}=W\left(:, 1: k_{f}\right)
$$

The columns of these matrices form the bases which are the solutions to (19) for the corresponding snapshot sets.

To complete the POD reduction, we define the reduced variable

$$
\mathrm{v}=\mathrm{U} \tilde{v}
$$

and upon substitution into (12) we arrive at the reduced-order system

$$
\tilde{v}^{\prime}=\mathrm{U}^{T} \mathrm{HU} \tilde{v}-\mathrm{U}^{T} \mathrm{~N}(\mathrm{U} \tilde{v}, \mathrm{w})+\mathrm{U}^{T} \mathrm{G} \cdot\left(\mathrm{U} \tilde{v}-\mathrm{E}_{s}\right) .
$$

3.2 Reduction of the Nonlinear Term via the Discrete Empirical Interpolation Method

While the dimension of $(22)$ is now $k_{v} \ll N$, the nonlinear term still depends on the full dimension $N$, which indicates that the system has not been truly reduced. For, the reduced voltage $\tilde{v}$ must be projected up by $\mathrm{U}$ to the full subspace before we can evaluate the nonlinear term, and the result must be projected back down to the reduced subspace by $\mathrm{U}^{T}$. We apply an empirical interpolation method to find a set of spatial interpolation points $\mathbf{z}=\left\{z_{i}\right\}_{i=1}^{k_{f}}$ from which the behavior of the full nonlinear term can be reconstructed, thus reducing the complexity of $\mathrm{N}$ to $k_{f} \ll N$ (Barrault et al., 2004). This method, originally described for use with finite elements, has been extended to our case of finite differences via the discrete empirical interpolation method (DEIM) as given by (Chaturantabut and Sorensen, 2009).

We begin with the basis $\mathrm{W}$ for the snapshot set $\mathrm{F}$ of nonlinear terms and seek a "maximally independent basis set" for W (Nguyen et al., 2008). The first interpolation point $z_{1}$ is the index of the entry of $\mathrm{W}_{1}$ with the largest magnitude. For $i=2, \ldots, k_{f}$ each point $z_{i}$ is chosen as the index of the entry of the largest magnitude of the residual 


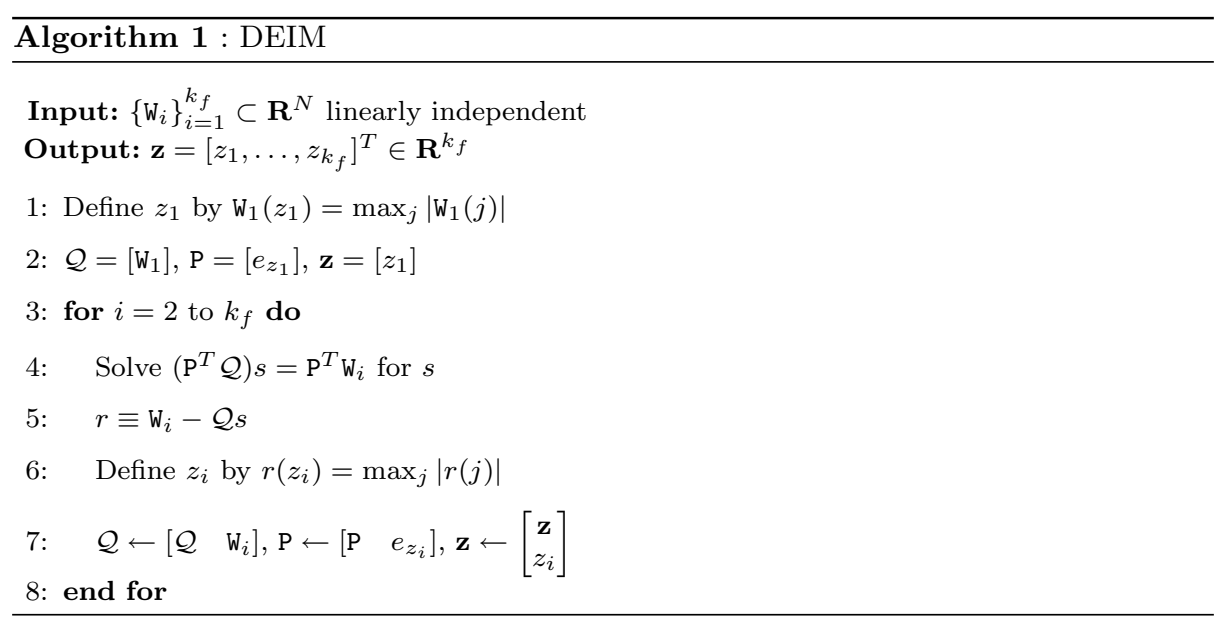

vector $r \equiv \mathrm{W}_{i}-\mathcal{Q} s$, where $\mathcal{Q}$ is the matrix of the previous $i-1$ DEIM basis vectors for $\mathrm{N}$ as given in Algorithm 1 (Chaturantabut and Sorensen, 2009).

We define the reduced gating variables

$$
\mathrm{w}=\mathrm{P} \tilde{w},
$$

substitute into the nonlinear term, and apply the matrices computed from the DEIM to obtain

$$
\mathrm{N}(\mathrm{U} \tilde{v}, \mathrm{w}) \approx \mathcal{Q}\left(\mathrm{P}^{T} \mathcal{Q}\right)^{-1} \mathrm{P}^{T} \mathrm{~N}(\mathrm{U} \tilde{v}, \mathrm{P} \tilde{w}) .
$$

Substituting (23)-(24) into (22) and (13), and applying (20), we obtain

$$
\begin{aligned}
\tilde{v}^{\prime} & =\tilde{\mathrm{H}} \tilde{v}-\mathrm{R} \tilde{\Phi}(\tilde{w})\left(\mathrm{e} \cdot \mathrm{Z} \tilde{v}-\mathrm{E}_{i}\right)+\mathrm{U}^{T} \mathrm{G} \cdot\left(\mathrm{U} \tilde{v}-\mathrm{E}_{s}\right) \\
\tilde{w}^{\prime} & =(\tilde{\mathrm{A}}(\tilde{v})-\tilde{w}) \cdot / \tilde{\mathrm{B}}(\tilde{v}),
\end{aligned}
$$

where the reduced functions are

$$
\tilde{\mathrm{A}}(\tilde{v}) \equiv \mathrm{P}^{T} \mathrm{~A}(\mathrm{U} \tilde{v}), \quad \tilde{\mathrm{B}}(\tilde{v}) \equiv \mathrm{P}^{T} \mathrm{~B}(\mathrm{U} \tilde{v}), \quad \text { and } \quad \tilde{\Phi}(\tilde{w}) \equiv \mathrm{P}^{T} \Phi(\mathrm{P} \tilde{w})
$$

and

$$
\begin{aligned}
\mathrm{Z} & \equiv \mathrm{P}^{T} \mathrm{U}: \mathbf{R}^{k_{v}} \rightarrow \mathbf{R}^{k_{f}} \\
\tilde{\mathrm{H}} & \equiv \mathrm{U}^{T} \mathrm{HU}: \mathbf{R}^{k_{v}} \rightarrow \mathbf{R}^{k_{v}} \\
\mathrm{R} & \equiv \mathrm{U}^{T} \mathcal{Q}\left(\mathrm{P}^{T} \mathcal{Q}\right)^{-1}: \mathbf{R}^{k_{f}} \rightarrow \mathbf{R}^{k_{v}} .
\end{aligned}
$$

Since in (27) all are pointwise functions, the matrix $\mathrm{P}^{T}$ just picks off the entries at the interpolation points $\mathbf{z}$, and thus by recalling (14) we find

$$
\tilde{\mathrm{A}}(\tilde{v})_{i} \equiv w_{:, \infty}\left((\mathrm{Z} \tilde{v})_{i}\right), \quad \tilde{\mathrm{B}}(\tilde{v})_{i} \equiv \tau_{:}\left((\mathrm{Z} \tilde{v})_{i}\right), \quad i=1, \ldots, k_{f}
$$

and similarly $\tilde{\Phi}$ just computes the rows of $\Phi$ corresponding to the indices $\mathbf{z}$. Hence the reduced functions are of complexity $k_{f}$, as desired. 
We solve the reduced system using the same staggered Euler scheme. We denote

$\mathrm{G}^{(j)}=\mathrm{G}((j-3 / 2) \Delta t), \quad \tilde{w}^{(j)} \approx \tilde{w}((j-3 / 2) \Delta t) \quad$ and $\quad \tilde{v}^{(j)} \approx \tilde{v}((j-1) \Delta t), \quad j=1,2, \ldots$ and use the scheme: Given $\tilde{w}^{(j-1)}$ and $\tilde{v}^{(j-1)}$, evaluate

$$
\tilde{w}^{(j)}=\left[\left(2 \tilde{\mathrm{B}}\left(\tilde{v}^{(j-1)}\right)-\Delta t\right) \cdot \tilde{w}^{(j-1)}+2 \tilde{\mathrm{A}}\left(\tilde{v}^{(j-1)}\right) \Delta t\right] \cdot /\left[2 \tilde{\mathrm{B}}\left(\tilde{v}^{(j-1)}\right)+\Delta t\right]
$$

and

$$
\tilde{v}^{(j)}=2 \tilde{v}_{\text {mid }}-\tilde{v}^{(j-1)}
$$

where, for current injection, $\tilde{v}_{\text {mid }}$ is the solution to the linear system

$$
\mathrm{L}^{(j)} \tilde{v}_{\text {mid }}=\mathrm{r}^{(j)}-\mathrm{U}^{T}\left(\mathrm{G}^{(j)} \cdot\left(\overline{\mathrm{v}}-\mathrm{E}_{s}\right)\right)
$$

where

$$
\begin{aligned}
& \mathrm{L}^{(j)} \equiv 2 I / \Delta t-\tilde{\mathrm{H}}+\operatorname{Rdiag}\left(\tilde{\Phi}\left(\tilde{w}^{(j)}\right) \mathrm{e}\right) \mathrm{Z} \\
& \mathbf{r}^{(j)} \equiv 2 \tilde{v}^{(j-1)} / \Delta t+\mathrm{R} \tilde{\Phi}\left(\tilde{w}^{(j)}\right) \mathrm{E}_{i} .
\end{aligned}
$$

In the case of synaptic conductance, $\tilde{v}_{\text {mid }}$ is the solution to the linear system

$$
\left(\mathrm{L}^{(j)}+\mathrm{U}^{T} \operatorname{diag}\left(\mathrm{G}^{(j)}\right) \mathrm{U}\right) \tilde{v}_{\text {mid }}=\mathrm{r}^{(j)}+\mathrm{U}^{T}\left(\mathrm{G}^{(j)} \cdot \mathrm{E}_{s}\right) .
$$

\subsection{Numerical Solution of the Reduced System}

We have written a MATLAB suite of functions that loads morphology and channel kinetics and distributions, simulates the full system and obtains POD and DEIM snapshots, and then simulates the reduced system and displays the response of these models to the desired inputs. Two ion channel models were used in this study: one follows Hodgkin-Huxley kinetics, while the other augments this model by including an A-type $K^{+}$current with a spatially-varying conductance density (we refer to these models as $\mathrm{HH}$ and HHA, respectively). Parameters for these models are the same as given in (Kellems et al., 2009). Gating variable evaluation was performed via a look-up table similar to (Hines, 1984). These codes are available from the authors upon request. All computations were performed on a Sun Ultra 20 computer with a $2.2 \mathrm{GHz}$ AMD Opteron processor. Detailed simulation documentation can be found in Supplementary Material 1.

\subsubsection{Computational Notes}

For the purpose of analyzing spiking behavior, we need only consider the voltage traces from one site (namely, the soma). It is easy to obtain this voltage $v_{\text {soma }}$ from the reduced system via (21), but computing the full matrix-vector product at each time step becomes expensive. However, noting that $\mathrm{v}_{\text {soma }}=\mathrm{U}\left(j_{\text {soma }},:\right) \tilde{v}$, it is clear that performing this inner product is much cheaper. Thus we can accelerate the reduced system by saving voltage data at different temporal resolutions: a fine resolution for the soma, and a coarse one everywhere else. For instance, saving the somatic potential every $r_{s}$ timesteps and saving all other potentials every $r_{c}$ timesteps, where $r_{s}<r_{c}$, will reduce the computational expense of (21) while still giving the detail we desire at the soma. In this paper, all timings reflect the use of $r_{s}=1$ and $r_{c}=10$. 


\section{Results on Simplified Morphologies}

\subsection{Straight Fiber}

Consider a uniform fiber that is $1 \mathrm{~mm}$ long with $N=1401$ compartments with HH kinetics. We generate a reduced model using 200 snapshots over $10 \mathrm{~ms}$ (solving (15)-(18) with $\Delta t=0.01 \mathrm{~ms}$ ) for both the POD and DEIM bases by applying a suprathreshold step current of $500 \mathrm{pA}$ for $1 \mathrm{~ms}$ at the distal end. This choice of stimulus location permits the action potential to traverse the whole fiber, thus providing a sufficiently rich set of snapshots from which to build the reduced system (see Figure 2A). Computing the singular values for the POD and DEIM snapshot matrices as in (3.1), we find that they decay quite rapidly (see Figure 2B), thus it is reasonable to expect that a small reduced system can approximate the full system well.
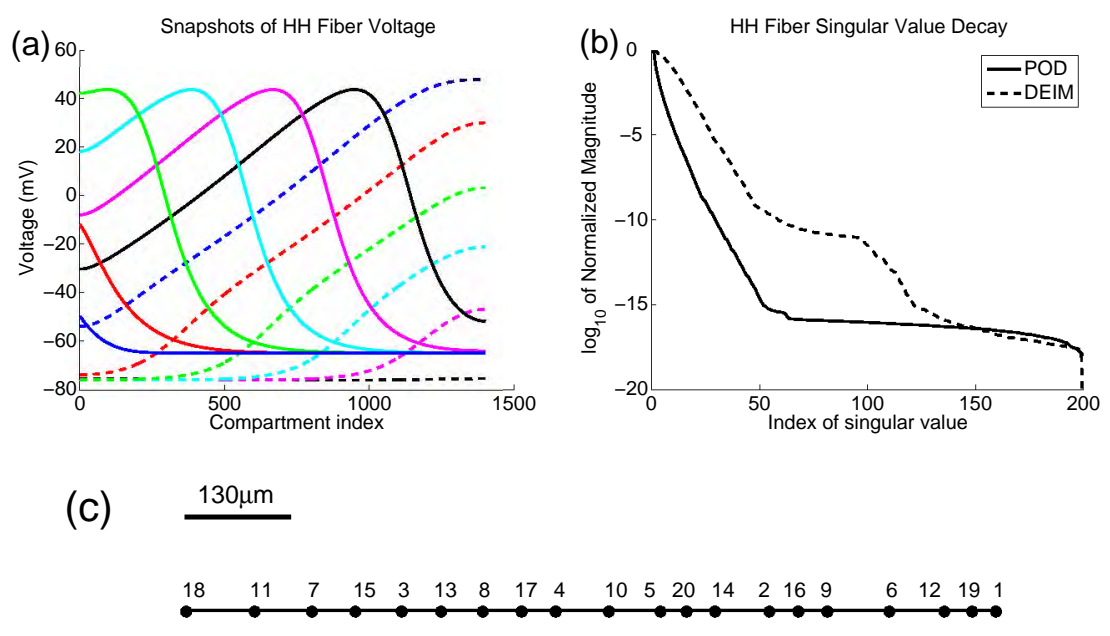

Fig. 2 (a) Sample traces of voltage snapshots used to construct the HH fiber reduced system. Shown are snapshots 22:10:132 of the simulation. (b) Decay of singular values for the POD and DEIM bases for the HH fiber. (c) Location of the first 20 DEIM points on the fiber, indexed by order in which they were computed

Note that the decay of these singular values to some tolerance does not necessarily imply a corresponding accuracy of the reduced system as compared to the full one. For example, for linear systems there exist SVD-based model reduction methods, such as Balanced Truncation, for which rigorous error bounds exist in terms of the decay of Hankel singular values (Glover, 1984) (Antoulas and Sorensen, 2001); these have been successfully applied to the linearized version of (1) (Kellems et al., 2009). For nonlinear systems the reduction relies upon having a good snapshot set. Hence if the snapshot set is poor, then the decay of the singular values cannot be interpreted as yielding a good reduced system. However, if the snapshot set is good then it can be used as a guide to estimate how large the reduced system must be.

To test accuracy and speed, we perform a series of simulations using random inputs and compare the reduced system's performance with that of the full system. A match is said to occur when a spike in the reduced system occurs within $\tau_{\text {ref }}=2 \mathrm{~ms}$ of a 
spike in the full system. Our metrics for quantifying the spike-capturing accuracy of the reduced system include the coincidence factor $\boldsymbol{\Gamma}$ (Kistler et al., 1997), defined as

$$
\boldsymbol{\Gamma}=\frac{N_{\text {match }}-N_{\text {full }} N_{\text {reduced }}\left(\tau_{\text {ref }} / T\right)}{\left(N_{\text {full }}+N_{\text {reduced }}\right)\left(1-N_{\text {full }}\left(\tau_{\text {ref }} / T\right)\right) / 2},
$$

where $N_{\text {full }}$ and $N_{\text {reduced }}$ are the number of spikes in the full and reduced models, respectively, $N_{\text {match }}$ is the number of matches, and $T$ is the length of the simulation. The value $\boldsymbol{\Gamma}=1$ implies the spike trains are equal and $\boldsymbol{\Gamma}=0$ implies the spike trains would occur purely by chance. To better evaluate the effect of false positives, we use the percentage matched and percentage mismatched measures of (Kellems et al., 2009):

$$
\begin{aligned}
\% \text { matched } & =100 \frac{N_{\text {match }}}{N_{\text {full }}} \\
\% \text { mismatched } & =100 \frac{N_{\text {reduced }}-N_{\text {match }}}{N_{\text {reduced }}} .
\end{aligned}
$$

We simulate for 1 second with 200 random step currents, each lasting 0-5 ms and having amplitudes of $0-100 \mathrm{pA}$, applied to random locations on the fiber. To solve both systems we use $\Delta t=0.1 \mathrm{~ms}$. We vary the dimension of the reduced system, using $k_{v}=k_{f}$, and run 20 simulations at each value (the same 20 input patterns were used for each reduced system). The results, shown in Table 1, indicate that reduced systems nearly 100 -fold smaller than the original ones reproduce highly accurate spiking patterns and are about $5 \times$ faster.

Table 1 Performance of reduced model (here $k_{v}=k_{f}$ ) of HH fiber, $N=1401$, as compared with the full model

\begin{tabular}{ccccc}
\hline$k_{v}$ & Speed-up & \% Matched & \% Mismatched & $\boldsymbol{\Gamma}$ \\
\hline 10 & $6.3 \times$ & 87.4 & 8.2 & 0.893 \\
15 & $5.9 \times$ & 98.9 & 1.1 & 0.988 \\
20 & $5.6 \times$ & 99.7 & 0 & 0.998 \\
30 & $4.6 \times$ & 100 & 0.6 & 0.997 \\
\hline
\end{tabular}

To assess the effects of different ion channel models on accuracy and speed, we perform the same experiment, but with a channel model incorporating an A-type $\mathrm{K}^{+}$ current (HHA model). Due to the decreased excitability of the distal part of the fiber, we increase the number of stimuli to 500 and the range of amplitudes of the step currents to 0-300 pA. As shown in Table 2, although the accuracy initially decreases for very small $k_{v}$ values, it is rapidly regained so that nearly exact spike dynamics $(\boldsymbol{\Gamma}=0.990)$ are reproduced with $k_{v}=k_{f}=30$, a 46 -fold reduction in dimension. Additionally, the reduced systems show greater speed-ups versus the full systems than do the $\mathrm{HH}$ ones. Examination of voltage traces (Figure 3) shows that not only are the spike times correct, but in fact the sub- and suprathreshold voltage dynamics agree very well.

One may wonder why the speed-up is basically one order of magnitude while the dimension reduction is two orders of magnitude. The reason lies in the fact that the full system matrices are large but sparse, whereas the reduced matrices are small but dense. Hence the associated matrix-vector products have different computational costs, and we ought not expect the same scalings. 
Table 2 Performance of reduced model (here $k_{v}=k_{f}$ ) of HHA fiber, $N=1401$, as compared with the full model

\begin{tabular}{ccccc}
\hline$k_{v}$ & Speed-up & \% Matched & \% Mismatched & $\boldsymbol{\Gamma}$ \\
\hline 10 & $8 \times$ & 81.7 & 64.3 & 0.457 \\
15 & $7.5 \times$ & 77.7 & 7.7 & 0.840 \\
20 & $6.9 \times$ & 95.8 & 0.5 & 0.976 \\
30 & $5.8 \times$ & 98.6 & 0.5 & 0.990 \\
\hline
\end{tabular}

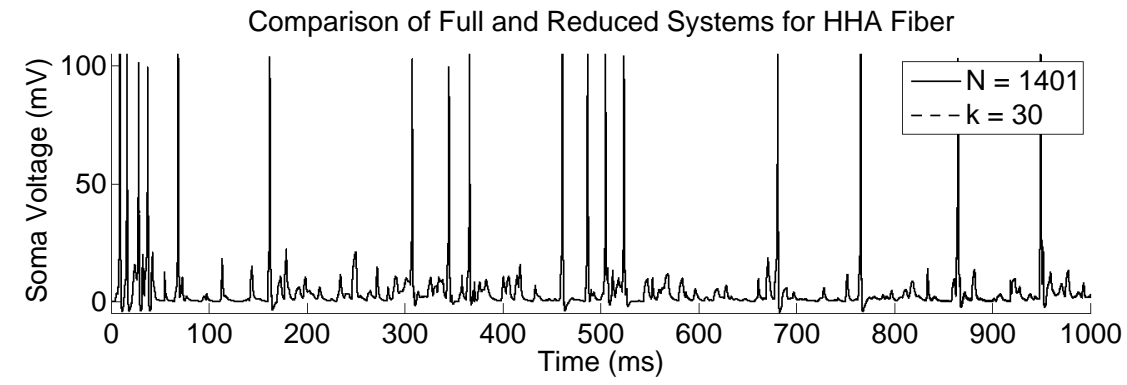

Fig. 3 Comparison of somatic voltage traces as computed by the full (solid) and reduced (dashed) systems for the HHA fiber. Although two curves are plotted, only one is seen because the reduced system is so accurate $\left(k_{v}=k_{f}=30\right)$ that it nearly exactly reproduces the dynamics

\subsection{Forked Neuron: Challenges of Branched Cells}

Now consider the a forked neuron which has one mother branch and two daughter branches, all with radius $1 \mu \mathrm{m}$ and length $500 \mu \mathrm{m}$. The cell consists of $N=1501$ compartments having $\mathrm{HH}$ kinetics. The first step in constructing a reduced model is to generate a sufficiently rich set of snapshots, but this simple cell illustrates that the task is not trivial.

\subsubsection{Phantom Spiking}

We desire a reduced model which will reproduce the correct dynamics of the full model independent of the location of the inputs. In order to capture spiking behavior, this implies that the snapshots to be used must contain local descriptions of action potentials. For example, on the fiber we needed to ensure that each node experienced spike dynamics. This was accomplished by initiating a spike at one end and allowing it to travel to the other end (this includes the after-hyperpolarization as well).

For the forked neuron, isolating the local spiking behavior is not so simple because each spike that is initiated will split upon reaching the junction point, and thus at least two branches will contain a spike at the same time. Such a situation is not good, because the snapshots will contain information about both spikes simultaneously (Figure 4), causing phantom spiking. This phenomenon consists of stimulus arriving at one location, but, because of the double spikes in the snapshots, the same output is observed at a mirror location on another branch (see Figure 5).

There are at least two remedies for this. The first is to take more snapshots and use inputs that are chosen so that the resulting set of snapshots will eliminate phantom 

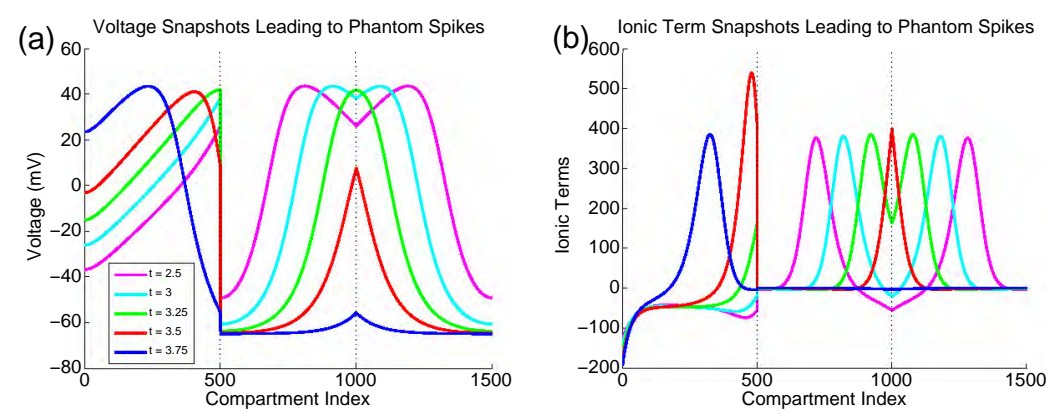

Fig. 4 The cause of phantom spiking can be traced to poor snapshots. Snapshots of (a) voltage and (b) ionic terms were obtained for the forked neuron during a $10 \mathrm{~ms}$ simulation in which the most distal node of branch 1 is stimulated with a $500 \mathrm{pA}$ step current lasting $1 \mathrm{~ms}$. Notice that the presence of two spikes (on different branches) occurs in both plots, but in this case it is more clearly shown in (b), which is used to generate the DEIM basis. Fine dotted lines in both plots indicate the ends of a branch

spiking. One such solution is to stimulate the extreme ends of each branch successively, so that each stimulus initiates a spike on one branch that then travels and splits onto the other two. In this way, there are snapshots in which each branch is active alone, and the effects of snapshots with two active branches cancel each other out. Though this approach works, it is not readily extensible to more complex morphologies. The forked neuron is essentially a symmetric morphology, so with some thought it is easy to conceive of a set of inputs that generate good snapshots. However, for a realistic hippocampal neuron it is not clear how to proceed, nor whether this approach has a solution.

\subsubsection{Branchwise Orthogonalization}

A more clever approach to solving the problem of phantom spiking is to try to exploit snapshots from a short simulation in order to tell us more about the local branch dynamics. The idea is to isolate the dynamics for a single branch and keep the other branches silent (i.e., at rest).

To accomplish this, we simulate the full model with a suprathreshold stimulus applied at a location which will allow a spike to propagate throughout the whole cell; this location should be the soma, a junction point, or the distal end of a leaf in order to prevent two spikes from occurring on the same branch. Let us denote the resulting set of snapshots as $\mathcal{S}$. To isolate the dynamics of branch $j$ we find the snapshots during which branch $j$ was active. We extract these snapshots from the original set to create a local set $\mathcal{L}_{j}$. These snapshots are then modified by setting to rest all the voltage values of elements that do not belong to branch $j$. The final new set of snapshots $\widehat{\mathcal{S}}$ is then the union of the local sets. Hence the snapshots are "orthogonal" in the sense that only one branch is active in each snapshot (Figure 6).

This technique, called Branch-Ortho, is formulated in Algorithm 2.

\subsubsection{V-Slim: Variance-based Snapshot Elimination Algorithm}

In order to implement the branchwise orthogonalization algorithm in any generality, we must have a notion of what it means for a branch to be active. The most obvious 


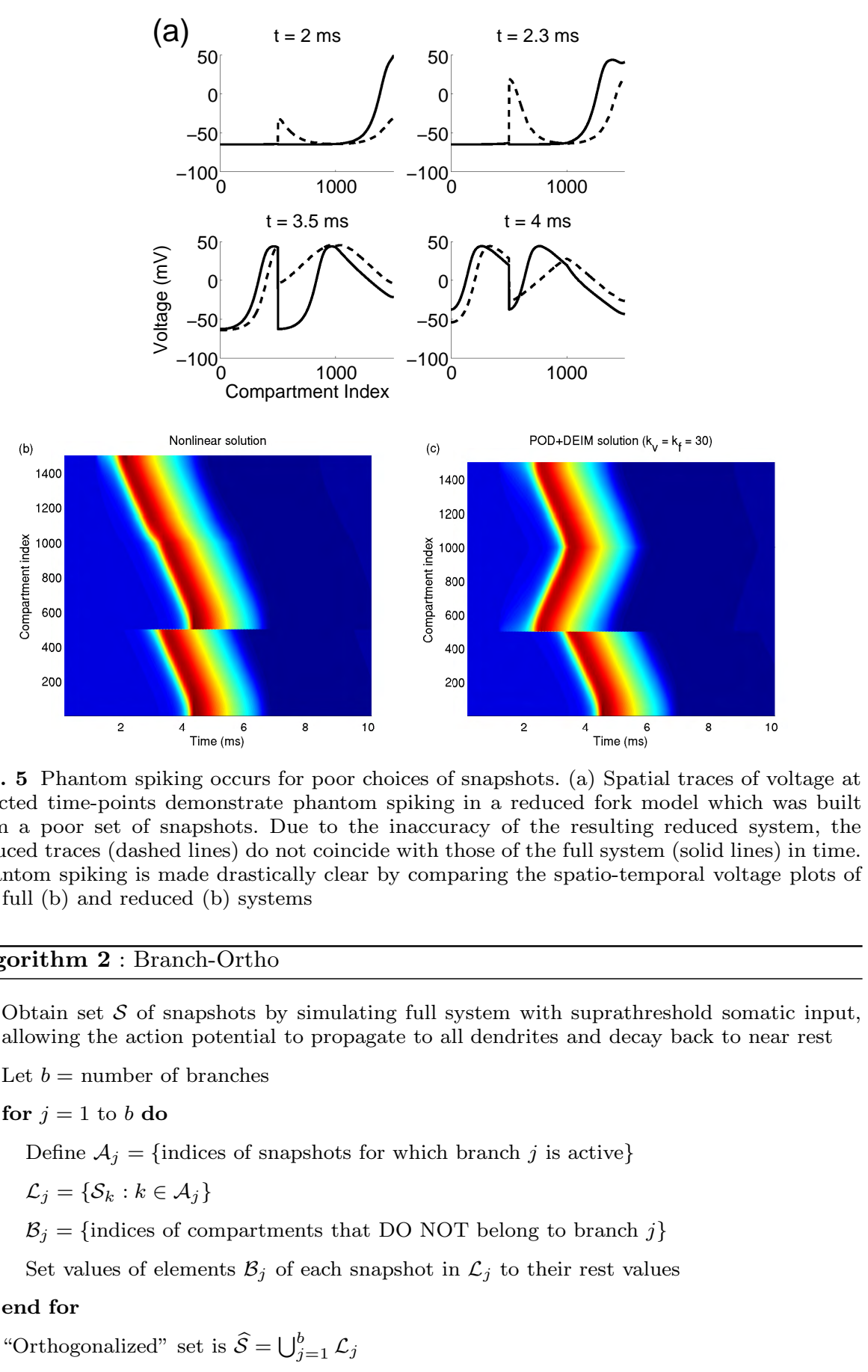



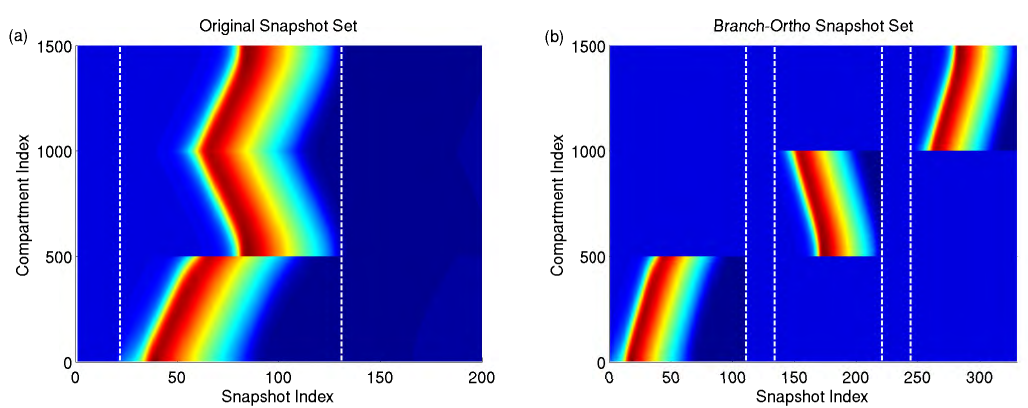

Fig. 6 Illustration of the Branch-Ortho and V-Slim algorithms. (a) The original set of snapshots, with white dashed lines bounding the global active zone (snapshots 22-131) obtained via the first application of V-Slim. (b) The resulting snapshot set after the Branch-Ortho algorithm is run, with white dashed lines enclosing the snapshots to be removed (111-134 and 221-244) as computed from the second application of V-Slim. The final snapshot set consists only of snapshots in the local active zones

notion is that the branch must not be at rest. However, since the cable equation is inherently diffusive, it is unlikely that under stimulation any branch will be exactly at rest. Yet, the change may be so small that it is insignificant. A more useful definition is that a branch is active when any compartment of that branch is "far enough" from rest.

A simple way to check this condition is to compute the variance with respect to rest of the snapshot and compare this value to the variances of the other snapshots, normalized to the snapshot with greatest variance. If this value is above some tolerance, then the branch is active; otherwise, the branch is inactive, and the snapshot can be neglected. This process, called V-Slim, is detailed in Algorithm 3.

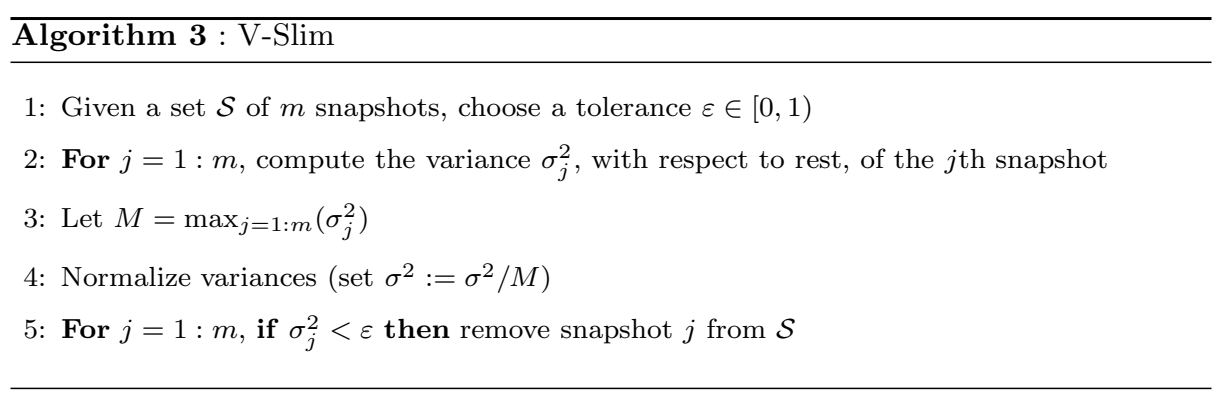

Another technique to shrink the size of $\widehat{\mathcal{S}}$ is to store only every $k$ th snapshot in $\mathcal{A}_{j}$. This is reasonable because snapshots which are close together in time are more similar. Keeping every snapshot gives maximal information, but we expect that we can get by with less (and we provide evidence in our results that this is indeed the case).

Such attention to shrinking the set of snapshots is necessary because computing the SVD's of the POD and DEIM inner product matrices is potentially very slow in our current implementation. Since the SVD scales as $\mathcal{O}\left(N^{3}\right)$, taking more snapshots will dramatically increase the computation time to obtain the reduced bases. For small numbers of snapshots $(\operatorname{dim}(\widehat{\mathcal{S}})<1000)$ this may not be so bad, but if we require 
many thousands of snapshots (as may be the case for highly-branched cells) then the SVD step may become intractable in a reasonable period of time. In the future we will use ARPACK (via the eigs command in MATLAB), which is a suite of iterative eigensolvers tailored to large-scale problems, to drastically reduce the cost of computing these SVD's. Previous experience gives us confidence that by using this package we can even eliminate the need for the aforementioned snapshot-shrinking algorithms. Nevertheless, the methods given here contribute a one-time cost, because results of these computations can be stored and reused for all future simulations of a specific morphology. This, along with the fact POD and DEIM set-up times for the simulations in this paper often required $\approx 1$ second and at most $\approx 10$ seconds, means we do not include set-up times in any of our timing comparisons.

\subsubsection{Branch-Ortho and V-Slim Implementation}

For branched cells it is useful to apply V-Slim to the orthogonalized set $\widehat{\mathcal{S}}$. In its first use in the Branch-Ortho algorithm, V-Slim operates globally in the sense that it determines the global active zone for the neuron. But for the orthogonalized set $\widehat{\mathcal{S}}$ all that matters is the local active zone, i.e. the snapshots for which a given branch is active. Since the local active zone is likely to be smaller than the global active zone, the difference is composed of inactive snapshots. By using V-Slim on $\widehat{\mathcal{S}}$ the method operates locally, pruning these artifacts (see Figure 6).

Thus our implementation of Branch-Ortho and V-Slim uses a 3-step process. First we apply V-Slim to $\mathcal{S}$ to obtain the global active zone, $\mathcal{A}_{\text {global }}$. Next we run Branch-

Ortho with $\mathcal{A}_{j}=\mathcal{A}_{\text {global }}$ to obtain $\widehat{\mathcal{S}}$. Finally, we apply V-Slim to $\widehat{\mathcal{S}}$, which effectively isolates the local active zones for each branch and yields the final snapshot set.

\subsection{Forked Neuron Results}

Equipped with the tools from the above sections, we return to the task of reducing the forked neuron. We take 200 snapshots over a $10 \mathrm{~ms}$ window (using $\Delta t=0.1 \mathrm{~ms}$ ), and we obtain them by giving a suprathreshold stimulus to a distal branch. We use the branchwise orthogonalization algorithm to generate snapshots of branches in isolation, where the active zone is computed by applying V-Slim with tolerances $10^{-6}$ and $10^{-5}$ for the POD and DEIM bases, respectively, and then saving every 4 th snapshot from this resulting set; no secondary V-Slim is used. Simulations consist of 750 random step currents injected over a 1 second interval, with each current having amplitude 0-60 pA and lasting $0-5 \mathrm{~ms}$.

Branch-ortho turns out to be a very effective method of improving the accuracy of the reduced system, as Table 3 demonstrates. Not only are spike times accurately reproduced, but the somatic voltage traces are nearly exactly duplicated in the reduced system, as shown in Figure 8. The improvement can be seen qualitatively in Figure 7 by observing that the DEIM points are more evenly spaced throughout the neuron than they are without Branch-Ortho.

Our next test is done with the HHA model, but with all the other parameters kept the same, except in this case, we use a secondary V-Slim algorithm with tolerances of $10^{-4}$ and $10^{-5}$ for the POD and DEIM bases, respectively, and we use 1000 random 
Table 3 Performance of reduced model (here $k_{v}=k_{f}$ ) of HH fork, $N=1501$, as compared with the full model. The first simulation set does not use Branch-Ortho for the reduced system, while the next four sets use it, leading to far superior accuracy

\begin{tabular}{lcccc}
\hline$k_{v}$ & Speed-up & \% Matched & \% Mismatched & $\boldsymbol{\Gamma}$ \\
\hline 30, no Branch-Ortho & $17.5 \times$ & 34.9 & 19.0 & 0.484 \\
\hline 10 & $24.1 \times$ & 63.4 & 16.0 & 0.718 \\
15 & $22.5 \times$ & 88.3 & 2.8 & 0.925 \\
20 & $21.2 \times$ & 94.3 & 0.8 & 0.967 \\
30 & $17.5 \times$ & 99.6 & 0.4 & 0.996 \\
\hline
\end{tabular}

stimuli of between 0-250 pA. Even though the spatially-varying A-type $K^{+}$conductance necessitates a slightly larger reduced system in order maintain the accuracy seen in the $\mathrm{HH}$ fork, we still observe a similar speed-up, as Table 4 indicates.

Table 4 Performance of reduced model (here $k_{v}=k_{f}$ ) of HHA fork, $N=1401$, as compared with the full model

\begin{tabular}{ccccc}
\hline$k_{v}$ & Speed-up & \% Matched & \% Mismatched & $\boldsymbol{\Gamma}$ \\
\hline 20 & $23.5 \times$ & 66.7 & 21.6 & 0.713 \\
25 & $22.0 \times$ & 97.3 & 2.9 & 0.971 \\
30 & $19.7 \times$ & 97.1 & 0.8 & 0.981 \\
40 & $15.4 \times$ & 98.7 & 0 & 0.993 \\
\hline
\end{tabular}

\subsection{Branched Cell Obeying Rall's 3/2 Power Law}

To examine more complex branching patterns we now consider a binary branching tree which satisfies the $3 / 2$ power law as explained in (Rall, 1959). The cell has a depth of 3 (for a total of 15 branches), and the root branch has length $200 \mu \mathrm{m}$ and radius $8 \mu \mathrm{m}$, agreeing with the morphological parameters as given in Rallpack 2 of (Bhalla et al., 1992). We consider two variants of such a tree: one in which the dendritic radii do not taper and one in which they do. We run 20 simulations of $1000 \mathrm{~ms}$ each with $\Delta t=0.1$ $\mathrm{ms}$, just as for the forked neuron, only this time we use 600 random currents of $0-500$ pA amplitude and 0-5 ms duration.

The results shown in Table 5 show that the reduced system recovers spiking dynamics to high accuracy. The tapered cell is less excitable than the non-tapered cell (which may be expected since there are more compartments with greater surface areas in the tapered cell). Also, the non-tapered cell requires a larger reduced system to capture the dynamics, which suggests that cells with smoothly varying dendritic radii may yield better results than cells whose radii change abruptly.

\section{Results on Realistic Morphologies}

Realistic cells necessitate simulations of larger dimension (to retain high spatial resolution for the inputs) and more POD and DEIM basis vectors (to maintain high accuracy 
(a)

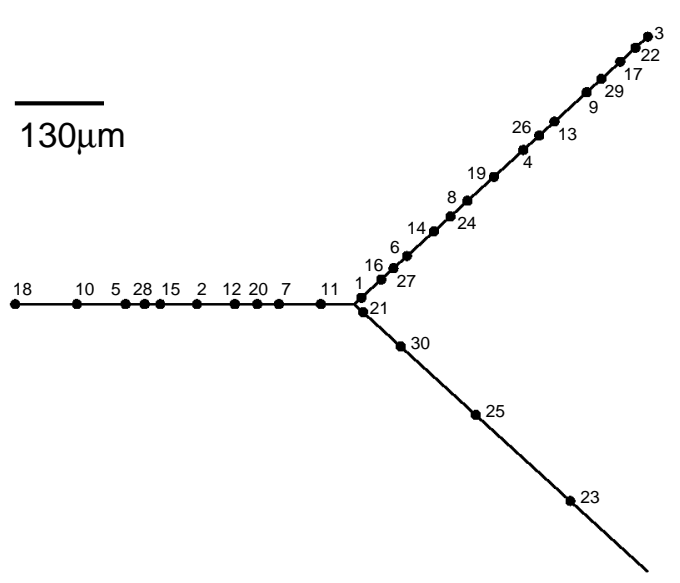

(b)

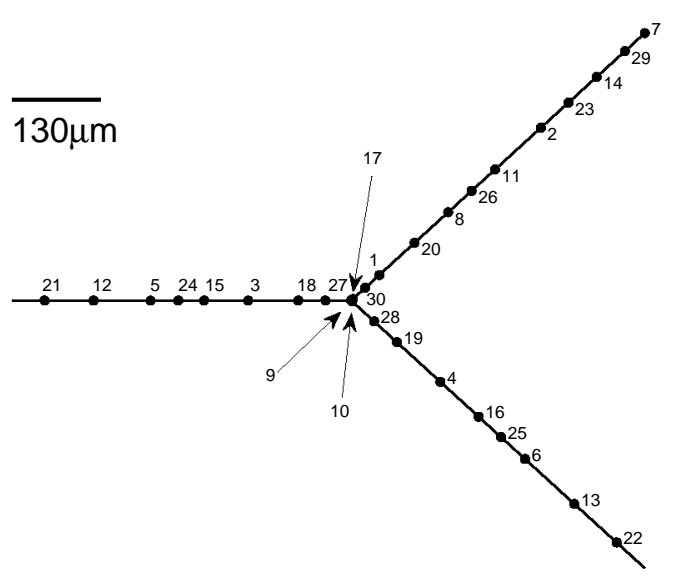

Fig. 7 DEIM points computed for the HH forked neuron when branchwise orthogonalization is (a) not used and (b) used. The improvement from using Branch-Ortho is evident in the qualitative difference in DEIM point location: notice that in (a) the lower-right branch has few DEIM points, whereas in (b) the DEIM points are more evenly distributed. In (b) there is a small cluster of points near the junction, with each point lying on distinct branches

Table 5 Performance of reduced model (here $k_{v}=k_{f}$ ) of HH Rall cells of depth 3 for tapered and non-tapered branches, $N=1823$, as compared with the full model

\begin{tabular}{lcccc}
\hline$k_{v}$ & Speed-up & \% Matched & \% Mismatched & $\boldsymbol{\Gamma}$ \\
\hline 30, tapered & $19.0 \times$ & 95.9 & 1.1 & 0.974 \\
30, non-tapered & $18.9 \times$ & 79.2 & 8.9 & 0.847 \\
40, non-tapered & $14.8 \times$ & 91.5 & 5.6 & 0.929 \\
50, non-tapered & $12.0 \times$ & 93.9 & 5.4 & 0.941 \\
60, non-tapered & $9.8 \times$ & 96.9 & 3.8 & 0.965 \\
\hline
\end{tabular}


(a)

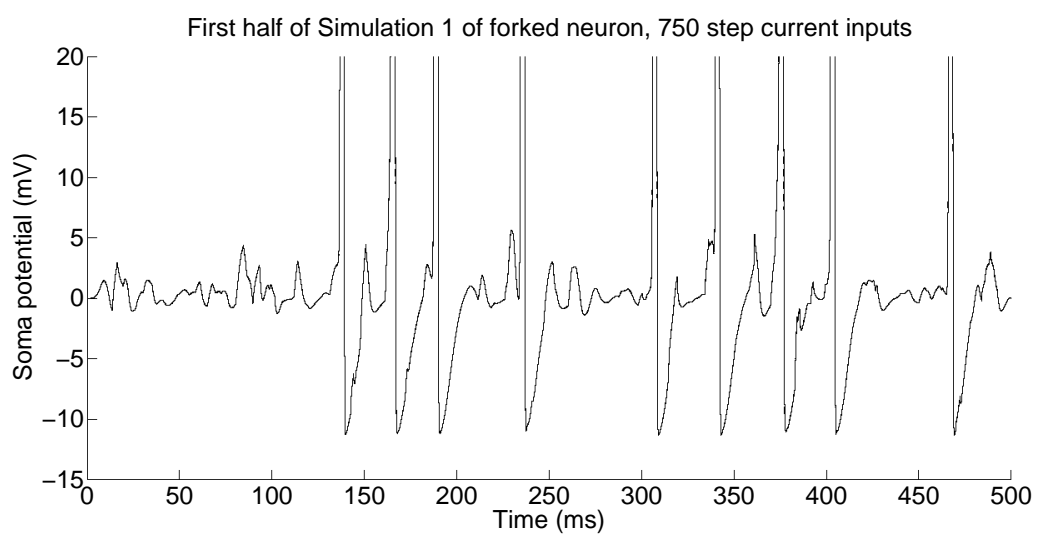

(b)

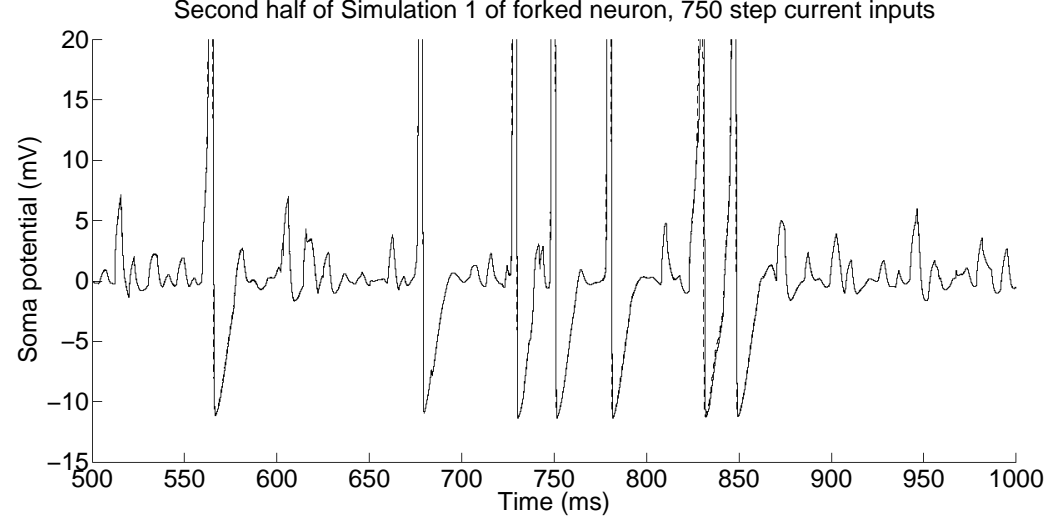

Fig. 8 Somatic voltage traces of the nonlinear and reduced models for (a) first half and (b) second half of simulation 1 of the $\mathrm{HH}$ forked neuron

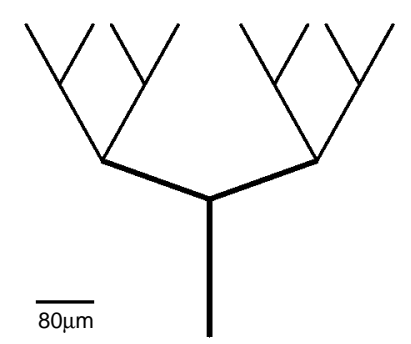

Fig. 9 Rendering of a binary branching cell of depth 3 obeying Rall's $3 / 2$ power law. The root branch has length $200 \mu \mathrm{m}$ and radius $8 \mu \mathrm{m}$, agreeing with the morphological parameters as given in Rallpack 2 of (Bhalla et al., 1992)

of the reduced system). There are many more dendrites on realistic cells than the toy neurons we considered in $\S 4$, and thus the branchwise orthogonalization will generate many snapshots that must then be pruned to obtain the most relevant ones in order to be able to compute the SVD in a reasonable time (as mentioned in $\S 4.2 .3$, the use 
of ARPACK may eliminate this bottleneck). This snapshot set $\widehat{\mathcal{S}}$ typically yields poor results on realistic cells because the branchwise orthogonalization algorithm in $\$ 4.2 .2$ completely isolates branches, effectively throwing away information about the coupling that occurs at junctions. Fortunately, this information can be partially recovered by considering a more intelligent variant of Branch-Ortho.

\subsection{Branch-Ortho with Routes}

A major improvement is to not just generate snapshots of isolated branches, but of connected branches that form continuous routes throughout the dendritic tree. A route $\mathrm{R}$ is defined as a set of branches in which

- at most one branch is present at each depth in the dendritic tree

- the branch at depth $j-1$ is the parent of the branch at depth $j$.

Hence the dimension of a route, $\operatorname{dim}(\mathrm{R})$, is equal to the number of branches it contains, and the length of a route is the sum of the lengths of the branches it contains, i.e. $\operatorname{len}(\mathrm{R})=\sum_{b \in \mathrm{R}} \ell_{b}$. Routes are useful because if we are given a set of snapshots in which an action potential travels from the soma throughout the dendritic tree, then routes form long, continuous snapshots of the dynamics and maintain coupling information between parents and children.

Not only does using routes improve accuracy, but it further shrinks the size of the snapshot set. Since the active zones of the parent and child branches overlap, routes yield more information in fewer snapshots (see Figure 10).

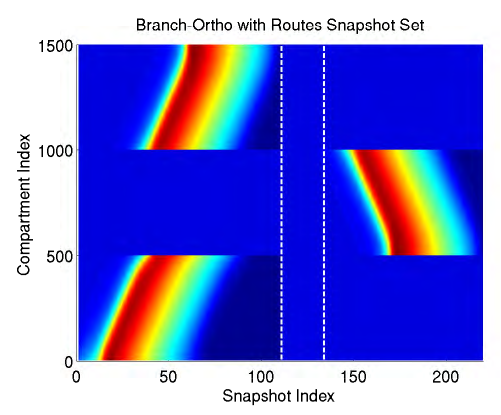

Fig. 10 Illustration of Algorithm 4 on a forked neuron. Two routes are used: one consisting of branches 1 and 3, and one of just branch 2. Notice that information about the coupling at the junction is preserved in the first route, and that fewer snapshots are used than in the original algorithm (compare with Figure 6(b)). White dashed lines in this plot enclose the snapshots (111-134) to be removed, as computed via the second V-Slim application

Which routes should comprise the Branch-Ortho snapshot set? Let $\mathcal{R}$ denote a set of routes and let $\operatorname{dim}(\mathcal{R})$ be defined as the number of routes in the set. We define $\mathcal{R}$ to be optimal if

- it contains every branch of the cell

- all routes are non-intersecting (i.e., no branch appears in more than one route)

- it contains the fewest routes possible, 
and we denote an optimal set of routes by $\widehat{\mathcal{R}}$. After a little thought, we can conclude that we can build an optimal set of routes entirely of routes which begin with leaves. Thus we know that if there are $L$ leaves in a cell, then we need to store only $L$ routes, and hence $\operatorname{dim}(\widehat{\mathcal{R}})=L$. Thus the smaller the ratio of $L / \mathcal{B}$ the better this method should be. It also follows that every $\widehat{\mathcal{R}}$ contains at least one $\mathrm{R}$ such that $\operatorname{dim}(\mathrm{R})=1$. Algorithm 4 implements Branch-Ortho with a set of optimal routes, which is obtained by proceeding outside-in from leaves to soma.

Is there a best set of routes? Probably, but we do not know it for certain. We merely conjecture that the best set of routes, $\mathcal{R}^{*}$, is the optimal one with the longest smallest route, i.e. it must maximize the length of the shortest route of dimension 1 :

$$
\mathcal{R}^{*}=\arg \max _{\widehat{\mathcal{R}}}\left[\min _{\substack{\mathrm{R} \in \widehat{\mathcal{R}} \\ \operatorname{dim}(\mathrm{R})=1}} \operatorname{len}(\mathrm{R})\right] .
$$

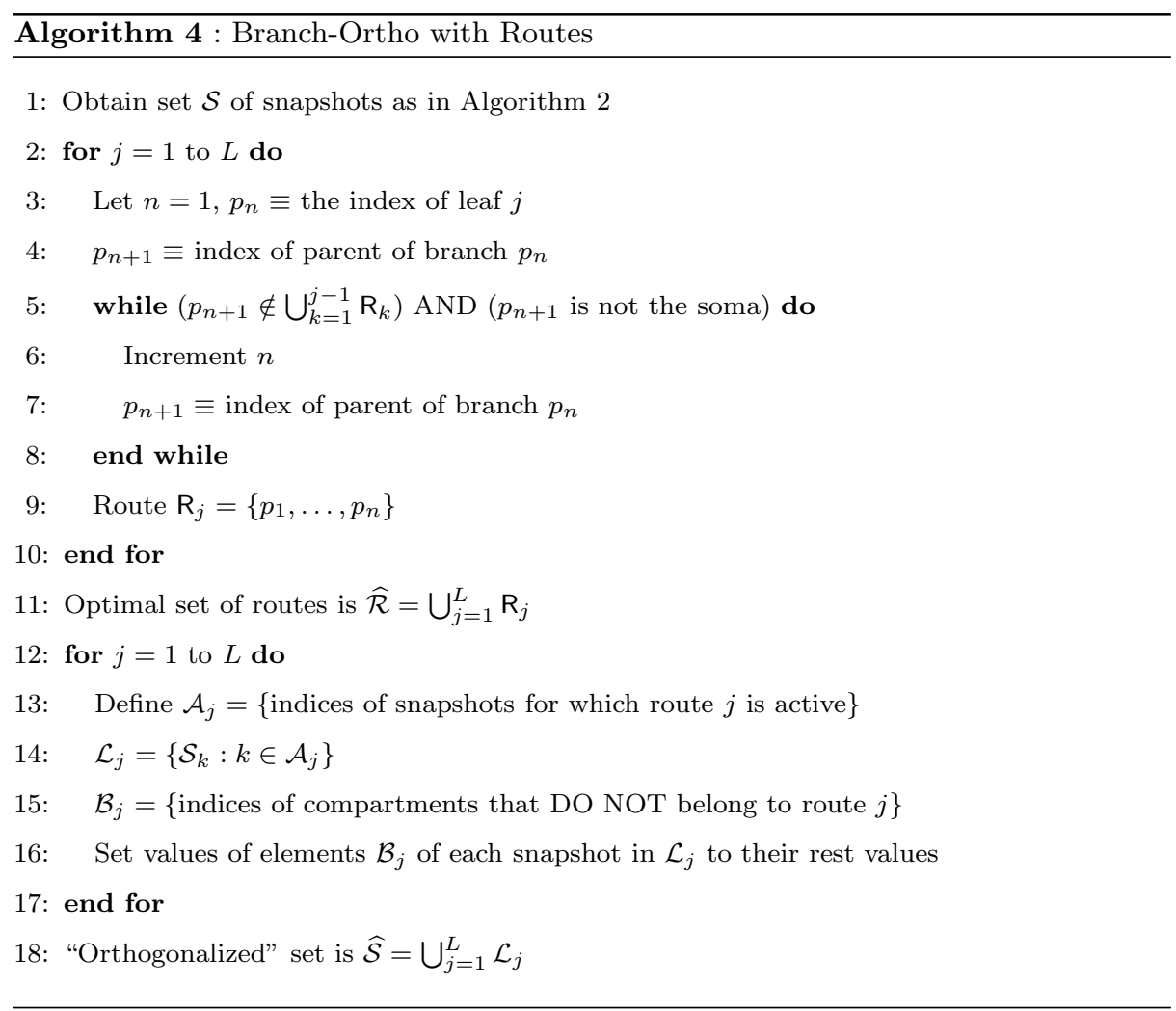

\subsection{Results on Neuron AR-1-20-04-A}

Consider the projection interneuron AR-1-20-04-A (Martinez), which has 35 branches and 20 leaves. At $1 \mu \mathrm{m}$ resolution, the full system has $N=2233$ compartments. 
We conduct a set of 20 simulations of 1 second duration, each using 500 random current injections of amplitudes between 0-150 pA and lasting 0-5 ms. We compare the spike-capturing accuracy of the full system to that of two reduced systems: one with routes, and one without. In both systems, the V-Slim tolerances are $\varepsilon_{v}=0.002$ and $\varepsilon_{f}=0.0005$, the active zone tolerances are $\epsilon_{v}=10^{-6}$ and $\epsilon_{f}=10^{-5}$, and the step between snapshots in the active zone is 4 . It should be noted that the original, un-orthogonalized snapshot set $\mathcal{S}$ is the same for both reduced systems; the only thing different is how we generate $\widehat{\mathcal{S}}$.

The dramatic improvement in accuracy is shown in Figure 11. Notice that this seemingly small change in the Branch-Ortho algorithm nearly doubles the accuracy. In fact, for $k_{v}=k_{f}=60$ the coincidence factor without routes is $\boldsymbol{\Gamma}=0.587$ while with routes it leaps to $\boldsymbol{\Gamma}=0.916$. Increasing the dimensions of the POD and DEIM bases from 60 to 90 , the coincidence factors reach $\boldsymbol{\Gamma}=0.953$ and 0.977 , respectively. The implications of these results are huge because smaller reduced systems can be constructed using Algorithm 4, decreasing the simulation time greatly.

(a)

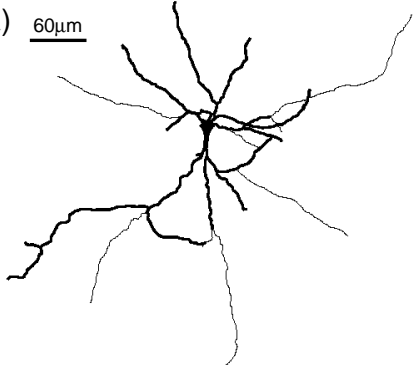

(b) Comparison of Accuracy of Reduced Systems (b) Using Branch-Ortho with and without Routes

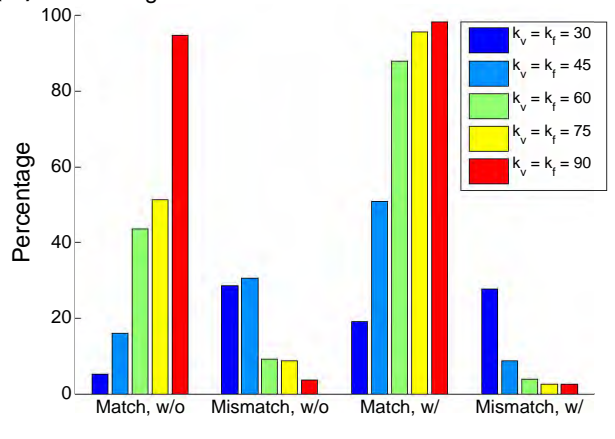

Fig. 11 (a) Rendering of cell AR-1-20-04-A (Martinez). (b) Bar plot showing the percentage matched and mismatched for the reduced systems (with and without routes) for cell AR-1-2004-A. Using routes dramatically increases the accuracy of the reduced system

Table 6 AR-1-20-04-A, $N=2233$ (here $k_{v}=k_{f}$ ), 500 step currents, HHA model, with and without routes

\begin{tabular}{lcccc}
\hline$k_{v}$ & Speed-up & \% Matched & \% Mismatched & $\boldsymbol{\Gamma}$ \\
\hline $30 \mathrm{w} / \mathrm{o}$ & $29.0 \times$ & 5.3 & 28.6 & 0.096 \\
$30 \mathrm{w} /$ & $29.0 \times$ & 19.2 & 27.7 & 0.299 \\
$45 \mathrm{w} / \mathrm{o}$ & $19.7 \times$ & 16.0 & 30.7 & 0.256 \\
$45 \mathrm{w} /$ & $19.7 \times$ & 50.8 & 8.9 & 0.649 \\
$60 \mathrm{w} / \mathrm{o}$ & $14.8 \times$ & 43.7 & 9.3 & 0.587 \\
$60 \mathrm{w} /$ & $14.8 \times$ & 87.9 & 4.0 & 0.916 \\
$75 \mathrm{w} / \mathrm{o}$ & $9.6 \times$ & 51.3 & 8.9 & 0.654 \\
$75 \mathrm{w} /$ & $9.6 \times$ & 95.5 & 2.7 & 0.963 \\
$90 \mathrm{w} / \mathrm{o}$ & $6.9 \times$ & 94.7 & 3.7 & 0.953 \\
$90 \mathrm{w} /$ & $6.9 \times$ & 98.1 & 2.6 & 0.977 \\
\hline
\end{tabular}




\subsection{Results on Other Morphologies}

The POD and DEIM modeling framework is applicable to a wide class of neurons comprising many brain regions. A growing online database (http://NeuroMorpho.org) (Ascoli, 2006) exists where experimentalists can upload detailed tracings of individual cells which may then be obtained by anyone and used in simulations. Using this database we have assembled a broad test group of neurons. In Table 7 we summarize the accuracy and speed of the corresponding reduced systems, and we plot the coincidence factors for a range of reduced system sizes in Figure 12. In general, the reduced systems are successful at capturing the spiking dynamics of each cell, and they simulate faster than their full system counterparts. Additionally, the voltage dynamics at any location can be recovered from the reduced system, not just the somatic potential, as illustrated in Figure 13. This offers convincing evidence that these model reduction techniques can be of use to a wide range of researchers in neuroscience.

Table 7 Model specifications and performance of reduced systems $\left(k_{v}=k_{f}\right)$ for the morphologies shown in Figure 12. Coincidence factors shown correspond to using Algorithm 4. Here $\mathcal{B}=$ number of branches in each cell

\begin{tabular}{llccccccc}
\hline Cell & Model & $\mathcal{B}$ & $h(\mu \mathrm{m})$ & $N$ & $k_{v}$ & $N / k_{v}$ & Speed-up & $\Gamma$ \\
\hline AR-1-20-04-A & HHA & 35 & 1 & 2233 & 75 & $29.8 \times$ & $9.6 \times$ & 0.963 \\
951005a & HHA & 44 & 1 & 1106 & 45 & $24.6 \times$ & $10.0 \times$ & 0.960 \\
pv22j & HH & 37 & 2 & 2686 & 75 & $35.8 \times$ & $10.2 \times$ & 0.751 \\
100103a & HHA & 32 & 1 & 2707 & 90 & $30.1 \times$ & $7.3 \times$ & 0.903 \\
mp_tb_40984_gc1 & HH & 54 & 1 & 2541 & 45 & $56.5 \times$ & $19.9 \times$ & 0.659 \\
512882 & HH & 35 & 2 & 2329 & 75 & $31.1 \times$ & $8.6 \times$ & 0.915 \\
P8-DEV66 & HH & 47 & 1 & 1712 & 60 & $28.5 \times$ & $9.8 \times$ & 0.903 \\
\hline
\end{tabular}

\section{Synaptic Conductances vs. Current Injection}

The reduced system can handle synaptic conductances and current injections, and they can be used interchangeably (i.e., a set of snapshots generated using current injection can also be used for a simulation with synaptic conductances). But, synaptic conductances take a computational toll on the reduced model because, in order to maintain an implicit time-stepping scheme, (31) implies that we must compute $\mathrm{U}^{T} \operatorname{diag}(\mathrm{G}(t)) \mathrm{U}$, where $\mathrm{G}(t)$ is the input synaptic conductance to each compartment. When many synaptic inputs are present, this becomes an expensive double-matrix product which can dominate the total simulation time. If alpha functions are used to model synaptic events, we can decrease the number of active inputs via a shutoff mechanism, thus greatly accelerating this computation.

Alpha functions can have a characteristic form

$$
g(t)= \begin{cases}g \frac{t-t_{\mathrm{on}}}{\tau} \exp \left(1-\frac{t-t_{\mathrm{on}}}{\tau}\right), & t<t_{\mathrm{off}} \\ 0, & t \geq t_{\mathrm{off}}\end{cases}
$$

which is always positive for $t \geq t_{\text {on }}$, where $\tau$ is the time constant and $\bar{g}$ is the maximal conductance. Here we assume that the synaptic event is inactive after some shutoff 
(a)

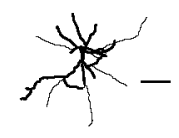

(b)

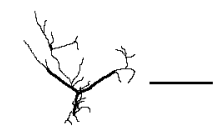

(c)

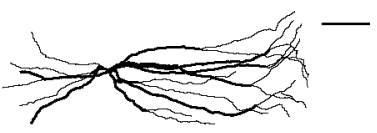

(d)

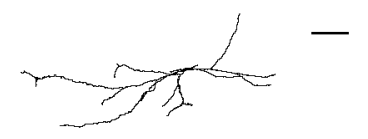

(e)

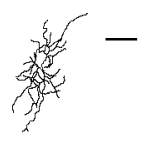

(f)

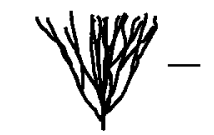

(g)

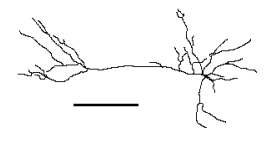

(h)
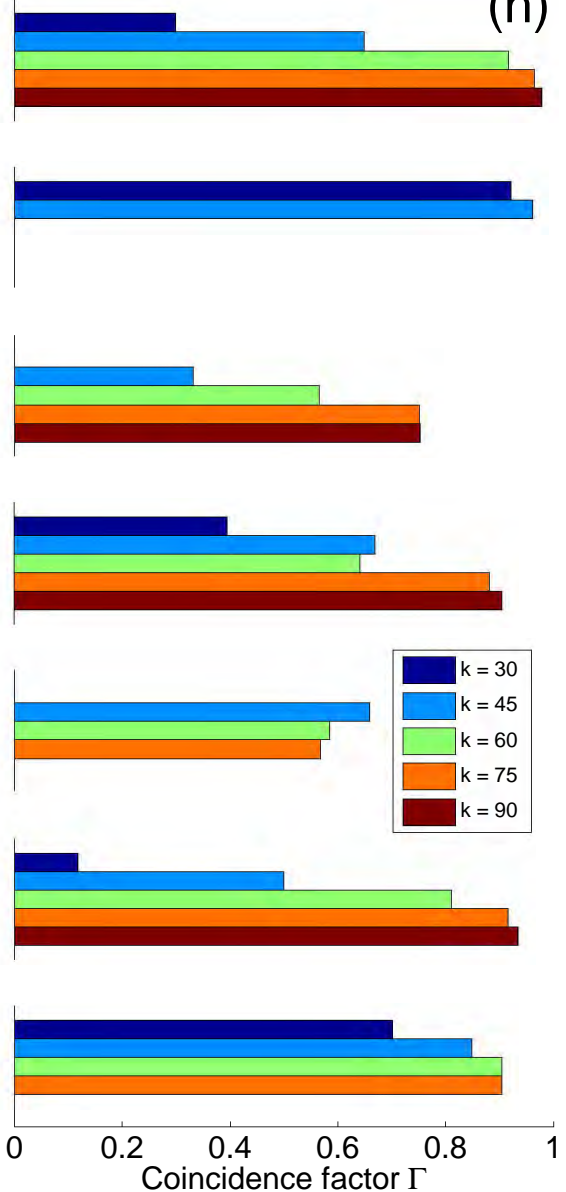

Fig. 12 Coincidence factors $\boldsymbol{\Gamma}$ show spike capturing accuracy for seven different cells using various sizes of reduced systems $\left(k=k_{v}=k_{f}\right)$ and Algorithm 4 (see (h)). From top to bottom the cells are: (a) projection interneuron AR-1-20-04-A (Martinez), (b) CA3 hippocampal interneuron 951005a (Chitwood et al., 1999), (c) CA1 hippocampal interneuron pv22j (Gulyás et al., 1999), (d) CA1 hippocampal interneuron 100103a from the stratum oriens (Golding et al., 2001) (Golding et al., 2005), (e) retinal ganglion cell mp_tb_40984_gc1 (Toris et al., 1995), (f) hippocampal cell 512882 from the dentate gyrus (Rihn and Claiborne, 1990), and (g) pyramidal cell P8-DEV66 from the cerebral cortex (Furtak et al., 2007). Scale bars on all cells represent $100 \mu \mathrm{m}$

time, $t_{\text {off }}$, at which $g\left(t_{\text {off }}\right)=\varepsilon$ for some small tolerance $\varepsilon$. Shutoff times for every alpha function can be computed via a rootfinding algorithm such as Newton's Method and stored in a queue for easy implementation during the simulation. 


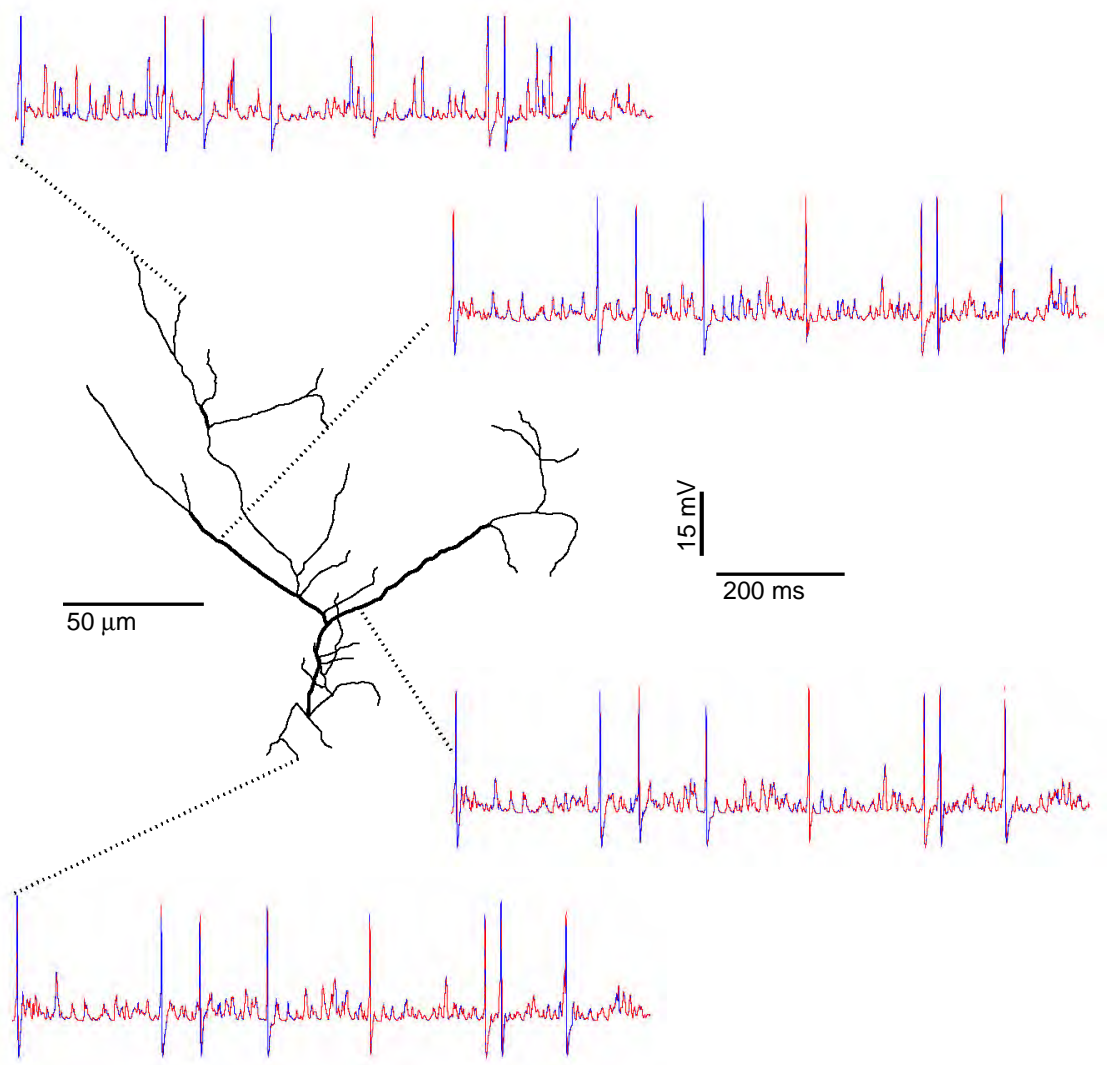

Fig. 13 Voltage traces for a few dendritic locations of the full $(N=1106$, blue $)$ and reduced $(k=45$, red) systems for simulation 1 of the cell in Figure 12(b). The traces are nearly identical for both systems, indicating that the reduced system can recover the global voltage dynamics to high accuracy. Only values below $30 \mathrm{mV}$ depolarizations are shown (i.e., when a spike occurs, any data point whose voltage was $>30 \mathrm{mV}$ from rest was omitted from the plot so that subthreshold dynamics could be more accurately shown)

Shutoff mechanisms can significantly speed up simulations when many synaptic inputs are used. For example, for cell AR-1-20-04-A with $N=2233, k_{v}=k_{f}=$ 60 , and 500 inputs with $\bar{g}=2 \mathrm{nS}$ and $\tau=1$, we observe a speed-up in calculating the double-matrix product from 30 seconds to 3.3 seconds when the shutoff tolerance $\varepsilon=10^{-4}$ is used. Now the reduced system is much more competitive with the full system, as Table 8 illustrates. Additionally, Table 9 shows that these systems provide accurate solutions, especially when the Branch-Ortho algorithm with routes is used. Compared to Table 6 the accuracy and speed-ups are less, due to the fact that synaptic input is more complex than current injection, but the reduced system is still very successful at capturing the cell's behavior. Finally, we note that shutoff mechanisms can be analogously implemented for any decaying synaptic conductance time course. 
Table 8 Alpha synapse shutoff mechanism $\left(\varepsilon=10^{-4}\right)$ accelerates the reduced system of neuron AR-1-20-04-A

\begin{tabular}{lcrr}
\hline \multirow{2}{*}{ Type } & Dimension & $\begin{array}{l}\text { Sim. Time (sec) } \\
\text { Without shutoff }\end{array}$ & With shutoff \\
\hline Full & $N=2233$ & 115 & 115 \\
Reduced & $k_{v}=k_{f}=60$ & 41 & 15 \\
Reduced & $k_{v}=k_{f}=90$ & 73 & 32 \\
\hline
\end{tabular}

Table 9 AR-1-20-04-A, $N=2233$ (here $k_{v}=k_{f}$ ), 500 alpha synapses, HHA model, with and without routes

\begin{tabular}{lcccc}
\hline$k_{v}$ & Speed-up & \% Matched & \% Mismatched & $\boldsymbol{\Gamma}$ \\
\hline $60 \mathrm{w} / \mathrm{o}$ & $8.9 \times$ & 21.2 & 41.5 & 0.302 \\
$60 \mathrm{w} /$ & $8.9 \times$ & 87.1 & 20.9 & 0.821 \\
$90 \mathrm{w} / \mathrm{o}$ & $4.6 \times$ & 93.0 & 22.7 & 0.834 \\
$90 \mathrm{w} /$ & $4.6 \times$ & 96.5 & 12.2 & 0.914 \\
\hline
\end{tabular}

\section{Discussion}

We have applied nonlinear model reduction techniques to morphologically realistic cells in a way that preserves the input-output relationships while accurately reproducing the complete voltage dynamics. We approximate the voltage using a POD basis, which reduces the number of state variables. Using the DEIM we build a set of spatial interpolation points and basis vectors to reduce the complexity of the nonlinear term so that it is proportional to the number of reduced variables. These techniques rely upon having "snapshots" of the dynamics which are rich enough to reproduce the full range of neuronal behavior. We introduce simple methods for obtaining these snapshots, and we develop algorithms to eliminate unimportant snapshots and to maximize the information in the important ones. When these tools are applied to realistic cells, they can generate reduced systems of dimensions nearly two orders of magnitude smaller than the originals and which yield highly accurate simulations in an order of magnitude less time.

Application of these model reduction techniques is likely to have the greatest effect on neuronal models with realistic ionic currents. Our simulations currently use standard, but limited, channel kinetics. Behaviors such as action potential initiation near the soma (Colbert and Pan, 2002) (Kole et al., 2008) rely upon better models for the spatial conductance densities, as well as the inclusion of different channels (Mainen and Sejnowski, 1998). Additionally, in this paper we have only considered excitatory inputs, but inhibition plays a key role in modulating neuronal output, both at the single cell and network levels (Brunel and Wang, 2003). Inhibition can be included with no change to the present methodology.

Synaptic conductances can also be treated via AMPA, GABA, and NMDA receptors, and their implementation in the reduced model is straightforward. The DEIM can even be used to reduce the dimension of the voltage-dependent NMDA receptor, as we have done in preliminary codes. But, for receptors to permit fast reduced simulations, we need to implement a shutoff mechanism similar to that in $\S 6$, which we have not done. 
An even higher level of realism can be achieved by modeling dendritic spines, which offers intriguing possibilities for the reduced system. Since spines interact only with their parent compartments, they have often been treated like gating variables for efficiency. However, this forces the use of hybrid time-stepping schemes, and also precludes consideration of multi-compartment spines, whose parameters such as spine head and spine neck sizes can affect the transmission of synaptic input (Schmidt and Eilers, 2009). Since the reduced systems are so small, we should be able to treat the spine compartments as true voltages, thus avoiding hybrid schemes and allowing true spatial coupling. Furthermore, if we assume each dendritic compartment has its own spine, then the full problem size grows to $2 N$ but the number of inputs remains at $N$, offering the promise of even greater dimension reduction and faster simulation.

A natural extension is to employ reduced cells in network simulations. Prototypical reductions can be computed for cells with different biophysical properties. These reduced cells are then building blocks that we can link together to form networks, opening the door to investigation of other neuronal functions, such as synaptic plasticity, using realistic morphologies but without the price of slow simulations.

Acknowledgements The work in this paper is supported by NSF grant DMS-0240058, by a training fellowship from the Keck Center for Interdisciplinary Bioscience Training of the Gulf Coast Consortia (NIBIB Grant No. 1T32EB006350-01A1), by AFOSR grant FA9550-061-0245, by AFOSR grant FA9550-09-1-0225, and by NSF grant CCF-0634902

\section{References}

A. C. Antoulas and D. C. Sorensen. Approximation of large-scale dynamical systems: An overview. International J. of Applied Math and Computer Science, 11(5):1093-1121, 2001.

G. A. Ascoli. Mobilizing the base of neuroscience data: the case of neuronal morphologies. Nature Reviews Neuroscience, 7:318-324, 2006.

M. Barrault, Y. Maday, N. C. Nguyen, and A. T. Patera. An 'empirical interpolation' method: application to efficient reduced-basis discretization of partial differential equations. $C . R$. Acad. Sci. Paris, 339:667-672, 2004.

U. S. Bhalla, D. H. Bilitch, and J. M. Bower. Rallpacks: a set of benchmarks for neuronal simulators. Trends in Neuroscience, 15(11):453-458, 1992.

N. Brunel and X.-J. Wang. What determines the frequency of fast network oscillations with irregular neural discharges? I. synaptic dynamics and excitation-inhibition balance. J. Neurophysiol., 90:415-430, 2003.

S. Chaturantabut and D. C. Sorensen. Discrete empirical interpolation for nonlinear model reduction. Technical Report TR09-05, Department of Computational and Applied Mathematics, Rice University, March 2009.

R. A. Chitwood, A. Hubbard, and D. B. Jaffe. Passive electrotonic properties of rat hippocampal CA3 interneurones. J. Physiol., 515:743-56, 1999.

C. M. Colbert and E. Pan. Ion channel properties underlying axonal action potential initiation in pyramidal neurons. Nat. Neurosci., 5:533-538, 2002.

S. C. Furtak, J. R. M. Jr., and T. H. Brown. Morphology and ontogeny of rat perirhinal cortical neurons. J. Comp. Neurol., 505(5):493-510, December 2007.

K. Glover. All optimal hankel-norm approximations of linear multivariable systems and their $L^{\infty}$-error bounds. Int. J. Contr., 39:1115-1193, 1984.

N. L. Golding, W. L. Kath, and N. Spruston. Dichotomy of action-potential backpropagation in CA1 pyramidal neuron dendrites. J. Neurophysiol., 86:2998-3010, 2001.

N. L. Golding, T. J. Mickus, Y. Katz, W. L. Kath, and N. Spruston. Factors mediating powerful voltage attentuation along CA1 pyramidal neuron dendrites. J. Physiol., 568:69-82, 2005.

A. I. Gulyás, M. Megías, Z. Emri, and T. F. Freund. Total number and ratio of excitatory and inhibitory synapses converging onto single interneurons and different types in the CA1 area of the rat hippocampus. J. Neurosci., 19:10082-10097, 1999. 
M. Hines. Efficient computation of branched nerve equations. International J. of Bio-Medical Computing, 15:69-76, 1984.

http://NeuroMorpho.org. The neuromorpho.org inventory. Accessed March 11, 2008. http: //NeuroMorpho.org.

A. R. Kellems, D. Roos, N. Xiao, and S. J. Cox. Low-dimensional, morphologically accurate models of subthreshold membrane potential. J. Comput. Neurosci., 2009.

T. B. Kepler, L. Abbott, and E. Marder. Reduction of conductance-based neuron models. Biological Cybernetics, 66:381-387, 1992.

W. M. Kistler, W. Gerstner, and J. L. van Hemmen. Reduction of the hodgkin-huxley equations to a single-variable threshold model. Neural Computation, 9:1015-1045, 1997.

M. H. P. Kole, S. U. Ilschner, B. M. Kampa, S. R. Williams, P. C. Ruben, and G. J. Stuart. Action potential generation requires a high sodium channel density in the axon initial segment. Nat. Neurosci., 11:178-186, 2008.

K. Kunisch and S. Volkwein. Galerkin proper orthogonal decomposition methods for a general equation in fluid dynamics. SIAM J. Numer. Anal., 40(2):492-515, 2002.

Y. C. Liang, H. P. Lee, S. P. Lim, W. Z. Lin, K. H. Lee, and C. G. Wu. Proper orthogonal decomposition and its applications-part I: Theory. J. Sound and Vibration, 252:527-544, 2002.

Z. F. Mainen and T. J. Sejnowski. Modeling active dendritic processes in pyramidal neurons. In C. Koch and I. Segev, editors, Methods in Neuronal Modeling: From Ions to Networks, pages 171-210. The MIT Press, 2nd edition, 1998.

J. O. Martinez. Rice-Baylor archive of neuronal morphology. Accessed May 1, 2008. http: //www. caam.rice.edu/ cox/neuromart.

N. C. Nguyen, A. T. Patera, and J. Peraire. A 'best points' interpolation method for efficient approximation of parametrized functions. Int. J. Numer. Meth. Engng., 73:521-543, 2008.

P. F. Pinsky and J. Rinzel. Intrinsic and network rhythmogenesis in a reduced Traub model for CA3 neurons. J. Comput. Neurosci., 1:39-60, 1994.

W. Rall. Branching dendritic trees and motoneuron membrane resistivity. Experimental Neurology, 1:491-527, 1959.

L. L. Rihn and B. J. Claiborne. Dendritic growth and regression in rat dentate granule cells during late postnatal development. Brain Res. Dev. Brain Res., 54(1):115-24, 1990.

H. Schmidt and J. Eilers. Spine neck geometry determines spino-dendritic cross-talk in the presence of mobile endogenous calcium binding proteins. J. Comput. Neurosci., 2009.

C. B. Toris, J. L. Eiesland, and R. F. Miller. Morphology of ganglion cells in the neotenous tiger salamander retina. J. Comp. Neurol., 352(4):535-59, 1995.

R. D. Traub and R. Miles. Neuronal Networks of the Hippocampus. Cambridge University Press, 1991. 